\title{
Global proteomic profiling of primary macrophages during $M$. tuberculosis infection identifies TAX1BP1 as a mediator of autophagy targeting
}

\author{
Jonathan M. Budzik ${ }^{1,2}$, Nick E. Garelis ${ }^{1}$, Teresa Repasy ${ }^{1}$, Allison W. Roberts ${ }^{1}$, Lauren M. \\ Popov $^{1}$, Trevor J. Parry ${ }^{3}$, David Jiminez-Morales ${ }^{4,5}$, Danielle L. Swaney ${ }^{5}$, Jeffrey R. Johnson ${ }^{5}$, \\ Nevan J. Krogan ${ }^{5}$, Jeffery S. Cox ${ }^{1}$ \\ ${ }^{1}$ Department of Molecular and Cell Biology, University of California, Berkeley, Berkeley, CA, \\ 94720 \\ ${ }^{2}$ Department of Medicine, Division of Pulmonary and Critical Care Medicine, University of \\ California, San Francisco, San Francisco, CA 94131 \\ ${ }^{3}$ Department of Microbiology and Immunology, Program in Microbial Pathogenesis and Host \\ Defense, University of California, San Francisco, San Francisco, CA 94158 \\ ${ }^{4}$ Department of Medicine, Division of Cardiovascular Medicine, Stanford University, CA 94305, \\ USA \\ ${ }^{5}$ Department of Cellular and Molecular Pharmacology, University of California, San Francisco, \\ San Francisco, CA 94158
}




\begin{abstract}
Macrophages are highly plastic cells that adopt diverse functional capabilities and play critical roles in immunity, cancer, and tissue homeostasis, but how these different cell fates and activities are triggered in response to their environmental cues is not well understood. We used new proteomic tools to identify protein post-translational modifications (PTMs) that control antibacterial responses of macrophages. Here, we report an unbiased and global analysis of the changes in host protein abundance, phosphorylation, and ubiquitylation, during the first 24-hours of Mycobacterium tuberculosis (Mtb) infection of primary macrophages. We discovered 1379 proteins with changes in their phosphorylation state and 591 proteins with changes in their ubiquitylation in response to $M t b$ infection. We identified pathways regulated by phosphorylation and ubiquitylation that weren't reflected by changes in protein abundance, indicating that the activity of these pathways was regulated. These include pathways known to be regulated by ubiquitylation and phosphorylation (e.g. autophagy) as well as pathways that were not known to be regulated during $M t b$ infection (e.g. nucleocytoplasmic transport and mRNA metabolism). We identified an enrichment in phosphorylation of autophagy receptors (TAX1BP1, p62, optineurin, BNIP3L), several of which were not previously implicated in the host response to $M t b$ infection. We found that p62 deficiency blocks ubiquitylation and TAX1BP1 deficiency enhances ubiquitylation, suggesting p62 ubiquitylation acts as an amplification loop by promoting downstream adaptor recruitment and serves as a platform for recruitment of ubiquitin. Our results show that TAX1BP1 mediates clearance of ubiquitylated $M t b$ and targets the bacteria to LC3-positive phagophores. Taken together, our proteomic profiling is likely a valuable resource for initiating mechanistic studies of macrophage biology.
\end{abstract}




\section{Introduction}

M. tuberculosis infection remains a major cause of mortality from infectious disease and drug resistant isolates pose a public health threat ${ }^{1-3}$. Our current understanding of the mechanisms by which $M t b$ is controlled by the immune system is only partially understood. While some pathways have been identified for $M t b$ control, there are likely many other pathways that await discovery. A better understanding of the immune mechanisms that underlie resistance to $M t b$ may shed light on new therapeutic and diagnostic modalities to combat this global health threat.

One of the modalities by which macrophages sense and respond to $M t b$ is by changing transcription $^{4,5}$, which leads to an increased pro-inflammatory state ${ }^{4,6}$. However, many other cellular processes (e.g. cell death pathways, autophagy, and trafficking) are not controlled by transcription $^{7,8}$. Understanding these non-transcriptional pathways will give us a more complete picture of the ways by which macrophages attempt to control Mtb infection and has revealed mechanisms by which intracellular pathogens persist in host cells ${ }^{9}$.

Autophagy is an example of a non-transcriptional macrophage response that is controlled both by phosphorylation and ubiquitin and is thought to lead to degradation of microbes in the lysosome ${ }^{10,11}$. Mtb perforation of the phagosomal membrane leads to activation of TANKbinding kinase 1 (TBK1), a kinase important for ubiquitin-mediated autophagy targeting as well as the induction of type I IFN ${ }^{12,13}$. Some ubiquitin ligases have been identified that are required for $M t b$-mediated autophagy ${ }^{14,15}$, but their substrates and role is still mysterious and there are likely other ones required. Deposition of ubiquitin subsequently recruit adaptors (p62, NDP52, and Nbr1) that link the ubiquitylated cargo to autophagy by the LC3-interacting region (LIR) ${ }^{16-}$ ${ }^{18}$. p62 has been shown to be phosphorylated and ubiquitylated in its ubiquitin binding domain, and both modifications have been shown to change the binding characteristics of the adaptor enabling recognition of polyubiquitylated cargo for selective autophagy ${ }^{19,20}$. In the case of $M t b$, some adaptors have been implicated in autophagy of the $M t b$-containing vacuole, but not all of them have been tested.

To improve our understanding of cellular responses to infection and especially autophagy, we profiled the changes in host protein abundance, phosphorylation, and ubiquitylation during the first 24-hours of $M t b$ infection in primary macrophages. By measuring dynamic changes in phospho-serine, -threonine, and -tyrosine with a label-free approach in primary cells during a dense time course of $M t b$ infection, we expand on our previously published work profiling changes in levels of post-translational modifications at a single time point in the RAW macrophage cell line ${ }^{21}$, and of tyrosine phosphorylation in primary macrophages ${ }^{22}$. Among the over 1,300 phosphorylated peptides that changed during $M t b$ infection, we identified significant changes in phosphorylation of the autophagy receptors p62, Bnip31, TAX1BP1, and Optineurin. We show for the first time that Optineurin and TAX1BP1 colocalize with $M t b$. Whereas ubiquitylation of $M t b$ is diminished in p62 targeted knockout macrophages, deficiency of TAX1BP1 results in accumulation of ubiquitylated $M t b$, and augments activation of TBK1 and recruitment of p62 to $M t b$. In contrast, $M t b$ colocalization with LC3 is inhibited in macrophages deficient for p62 or TAX1BP1. In this study, we systematically identified thousands of post- 
bioRxiv preprint doi: https://doi.org/10.1101/534917; this version posted January 31, 2019. The copyright holder for this preprint (which was not certified by peer review) is the author/funder. All rights reserved. No reuse allowed without permission.

translational modifications during macrophage infection, which enabled us to gain new insight in the distinct roles of autophagy receptors during $M t b$ infection. 


\section{Results}

\section{Proteome-level evaluation of primary macrophage responses to $M$. tuberculosis infection}

To identify new innate immune pathways modulated during $M$. tuberculosis infection, we sought to obtain a deep data set of the changes in host protein abundance and post-translational changes during a time course of macrophage infection. To this end, we infected primary murine bone marrow-derived macrophages with $M t b$ in biological triplicate, harvested infected cells at 2-, 4-, 6-, 8-, and 24-hours post-infection, and prepared protein lysates (Fig. 1A). We also performed exact time-matched mock infections of the same macrophages and harvested at 0-, 6-, and 24hours. Samples were digested with trypsin and a portion of the resulting peptides were set aside for abundance measurements and the remaining peptides were subjected to serial enrichment using di-gly remnant ${ }^{23}$ and phospho-peptide affinity technologies ${ }^{24}$. We subjected all peptide samples (abundance, phospho- and diGly-modified peptides) to liquid chromatography-mass spectrometry (LC-MS). Based on the measured $\mathrm{m} / \mathrm{z}$ ratio of the parent ions and fragment ions for each peptide coupled with a database search of the mouse proteome, we determined the amino acid sequence, modification sites, and full-length protein for each peptide. As an important quality control measure, pair-wise comparison of parent ion MS intensity measurements between technical and biological replicates from the same condition were highly reproducible with robust correlation coefficients from global abundance, phosphoenriched, or diGly-enriched samples (Table S1; Fig 1B).

To determine the statistically significant changes in protein abundance and/or PTMs in response to infection, we performed label-free quantification using MSstats to compare unfragmented (MS1) intensity peptide counts between infected and uninfected samples across the biological and technical replicates. Peptides with a $\log _{2}$-fold change in intensity of $>1$ or $<-1$ and a $p$ value $<0.05$ were deemed statistically significant. Importantly, in many cases, we identified significant peptide values in one experimental condition (infected) but no corresponding peptides in the cognate (uninfected) control, indicating that these changes were likely biologically relevant, but which complicated the computation of a meaningful ratio. To address this issue, we used a method described by Waters et al. in which missing values were imputed by the limit of MS1 detection to reveal an "imputed value" 25 . An aliquot of peptide samples were analyzed for changes in global protein abundance prior to enrichment for phosphopeptides or diGly-remnant peptides (Fig. 1A). Analysis of global protein abundance, phosphorylated peptides, and di-Gly remnant peptides revealed up to 50,000 peptides matching to 4,500 unique proteins (Supplemental S1) and correlating to over 1,000 statistically significant changes in peptide levels during Mtb infection (Supplemental S2, S3, and S4).

Over the 24-hour time course, we observed an increase in the number of changes in abundance and ubiquitylated proteins at each subsequent time point starting at 2-hours post-infection with the maximum number detected at 24-hours post-infection (Fig. 1C). In contrast, phosphopeptide changes were bimodal with the greatest number of phosphopeptide changes occurring early (2or 4-hours post-infection) or late (24-hours) after Mtb infection (Fig. 1C).

Although protein abundance levels are to a large extent determined by mRNA levels ${ }^{26}$, the correlation between the two is affected by the substantial delay (up to 6-hours) in protein 
abundance changes following changes in mRNA levels, post-translational modifications (i.e. ubiquitylation) affecting protein degradation, and translation rate modulation ${ }^{27}$. In order to determine the level of correlation between the changes in RNA and protein abundance after $M t b$ infection, we compared recently published transcriptomics dataset in murine bone marrowderived macrophages 24-hours post-infection with $M t b^{5}$ and transcriptomics datasets from our laboratory at 2-, 6-, and 24-hours post-infection. Overall there was modest correlation between protein and RNA abundance at 24-hours post-infection for proteins using non-imputed (rho=0.81) and imputed peptide methods (rho=0.78; Fig. 1D). Weaker correlations were observed between mRNA and protein abundance changes at 2- and 6-hours post-infection (Supplemental S5), or between transcriptional changes and phosphopeptide or ubiquitylated peptide levels (Fig. 1D). These results suggest that the change in RNA abundance is a moderate predictor for change in protein abundance and that post-translational mechanisms (i.e. ubiquitylation targeting proteins for degradation in the proteasome) play an important role in affecting protein abundance during $M t b$ infection.

\section{$M t b$ infection elicits unique and overlapping changes at the translational and post- translational level}

A complete list of changes in macrophage global protein abundance, phosphopeptide, and diGlyremnant peptides at 2-24-hours post-infection with $M t b$ are is found in Tables S4-S6. As an example, at 24-hours post-infection, the volcano plots highlight in green representative nonimputed peptides with statistically significant $p$ values $<0.05$ and a $\log _{2}$-fold change $>1$ or $<1$ (Fig. 2A). Imputed peptides were assigned a $p$ value just above the level of statistical significance and therefore are located immediately above the horizontal line demarcating the $p$ value of $0.5\left(-\log _{10}(p\right.$ value $)=1.3$; Fig $\left.2 \mathrm{~A}\right)$. Many of the proteins changing in global abundance and ubiquitylation, but to a lesser degree in their phosphorylation levels, are encoded by interferon- $\beta$ and $-\gamma$ regulated genes such as Ifit1, Ifit 2, Stat1, or Ifihl (Fig. 2A). When comparing the individual proteins at all of the time points changing in abundance $(n=470)$, phosphorylation $(n=1377)$, or ubiquitylation $(n=591)$ during $M t b$ infection, we again noted that there was limited overlap between proteins that changed in global abundance and those modified at the post-translational level (Fig. 2B).

Gene ontogeny classification is one annotation method by which groups of genes or proteins are characterized by overlapping cellular function and identify pathways involved in the host responses $^{28}$. To quantify how changes in individual protein or modification levels affect cellular pathways and gene ontogeny annotations, we performed a functional pathway meta-analysis with the web-based bioinformatics analysis pipeline, Metascape ${ }^{29}$. Among the 2,000 out of 9,381 gene ontogeny functional pathways enriched during infection $(\mathrm{p}<0.01), 296$ were shared between global abundance, phosphorylation, and ubiquitylation enrichment groups, and each group contained hundreds of overlapping proteins or proteins with the same ontology term (Fig. 2C). The most functional pathways changing during $M t b$ infection were identified by phosphorylation $(n=1412)$ followed ubiquitylation $(n=1111)$ and global abundance $(n=719$; Fig. $2 C)$.

Enrichment meta-analysis of gene ontogeny functional pathways that changed during Mtb infection from peptide changes pooled at all time points revealed enrichment for immunity, 
cellular trafficking, metabolism, and signal transduction pathways among the top 20 most statistically significant pathways (Fig. 2D). Unique to the phosphopeptide datasets was enrichment for small GTPase mediated signal transduction, whereas cellular transport pathway enrichment was revealed by global abundance and ubiquitylation changes (Fig. 2D).

\section{Changes in protein abundance reveal enrichment of antiviral and inflammatory pathways during $M$. tuberculosis infection}

We next sought to determine how the changes in protein abundance levels clustered together during the 24-hour time course of infection and the functional pathways represented by groups of proteins changing in the same direction following $M t b$ infection. Protein abundance and imputed protein changes each clustered into two large groups and several smaller clusters (Fig. 3A). The first group comprised of proteins increasing in abundance during $M t b$ infection and was functionally enriched for inflammatory, antiviral, and response to molecular of bacterial origin (Fig. 3B and Supplemental S6; $-\log _{10} p=12.66,24.60,14.08$ ). In contrast, the second cluster of proteins that decreased in abundance during $M t b$ and included ribosome biogenesis, DNA replication, and regulation of protein catabolism in the vacuole (Fig. 3B, Supplemental S6; $-\log _{10}$ $p=9.05,10.08,6.53)$. These findings are consistent with previous studies implicating a strong anti-viral response $\mathrm{e}^{30}$ and enrichment in ribosome biogenesis pathways in transcriptional changes following $M t b$ infection ${ }^{31}$

\section{Proteins ubiquitylated during $M$. tuberculosis infection enriched for immune responses and autophagy}

Ubiquitylated substrates clustered together into those decreasing during infection (Cluster 1, Fig. 4A), or those increasing early (2-4-hours; Cluster 2, Fig. 4A), mid (6-8-hours; Cluster 3, Fig. 4A), or late (24-hours; Cluster 4, Fig. 4A) post-infection. While the majority of the changes in functional pathways demonstrated the most statistically significant enrichment at 24-hours such as cellular protein catabolic process, response to virus, nicotinamide nucleotide metabolism, and cellular response to interferon gamma (Fig. 4A, cluster 4; $-\log _{10} p=13.21,10.01,8.30$ ), peptide metabolic process was a notable exception, which was most enriched at 2- and 4-hours postinfection (Cluster $2,-\log _{10} p=11.31$ ). Nucleosome assembly and actin cytoskeleton organization were the top two functional pathways that decreased in functional enrichment (Cluster $1,-\log _{10} p$ = 6.74, 6.23; Supplemental S6). Autophagy enrichment was most statistically significant at 6and 8-hours post-infection (Fig. 4A, cluster 3, $-\log _{10} p=8.09$ ). These findings are consistent with the notion that $M t b$, in addition to eliciting an antiviral response, affects nucleosome assembly, which has been directly implicated in viral pathogenesis and HIV gene transcription during $M t b$ co-infection ${ }^{32}$.

\section{Changes in host protein phosphorylation during M. tuberculosis infection impact cellular trafficking pathways}

Phosphopeptide changes during $M t b$ infection clustered into four groups: two groups increasing during infection at 2-4-hours (Fig. 5A, Class 1) or 6-24-hours (Class 2), and two groups decreasing during infection at 2-4-hours (Class 3) or 24-hours (Class 4). Functional enrichment for actin cytoskeleton organization was significant in all four clusters and especially in early in 
infection (Fig. 5A and B, Class $1,-\log _{10} p=15.29$ ). Additional cellular trafficking pathways changing during $M t b$ infection included endosomal, cytosolic, and nuclear transport (Fig. 5B). $M t b$ engaged signal transduction components including small GTPase and I- $\kappa \mathrm{B}$ kinase/NF- $\mathrm{BB}$ (Cluster 3, $-\log _{10} p=11.95$ and 9.29). This data suggests a prominent role for cellular trafficking pathways in the host response to $M t b$ infection at all of the measured time points and highlights the inflammatory signaling molecules activated during $M t b$ infection.

\section{Kinase prediction tools identified kinase families and ubiquitin ligase complexes changing during $M$. tuberculosis infection}

Although the functional significance of these phosphorylation changes await determination, bioinformatic tools that rely on the extremely large number of phosphoproteomic studies performed to date, allow for prioritization of those modification that likely regulate protein function and can infer when a specific kinase is regulated ${ }^{33}$. The large number of phosphorylation changes, and the temporal specificity, indicates that many kinases are activated during the 24-hour interaction of macrophages with $M t b$. To begin to identify these kinases, we used a bioinformatics approach to compare the substrates phosphorylated during Mtb infection with a database of known kinase substrate pairs. Interestingly, several potential kinases were identified from the earliest phosphorylation data, including GTF2F1, Aurora, and ITR, suggesting that these may be good targets for prioritization of signaling cascades activated early upon interaction. Indeed, Aurora kinase is interesting given its recently discovered relationship with HIV infection, and it has not been previous implicated in bacterial infection ${ }^{34}$. We also sought to predict the kinases involved in trafficking and motility (Fig. 6). Indeed, we predicted the activity of important kinases that regulate actin filament dynamics, rho-associated protein kinase 1 and its kinase substrates including LIMK1 and LIMK2, changed during the time course of $M t b$ infection (Fig. 6A). Consistent with the canonical role in JAK-STAT mediated activation of immune cell survival, activation, and recruitment and MAP2Ks in the stress-response, the kinase prediction tool revealed increased kinase activity of JAK as early as 2-hours postinfection and maximal levels of MAP2K activity at 24-hours post-infection (Fig. 6A). IKB kinase, another key inflammatory kinase involved in NF- $\kappa \mathrm{B}$ signaling ${ }^{35}$, was also predicted to undergo change in its kinase activity during $M t b$ infection.

Similarly, potential ubiquitin ligases can be prioritized by their presence in kinase complexes based on a bioinformatic search of the predicted kinase complexes from our phosphopeptide datasets. In addition to revealing the TRAF- 2 and -6 predicted ubiquitin ligase complexes, which have been previously implicated in immune signaling during infection ${ }^{36}$, the prediction tool identified previously unrecognized predicted ubiquitin ligase complexes including the ligases CUL3, RBX1, and MDM2 (Fig. 6B).

\section{p62, NBR1, optineurin, and TAX1BP1 colocalize with $M$. tuberculosis during macrophage infection}

The autophagy pathway was prominently enriched in both PTM datasets, and we noted a significant number of phosphorylation events that occurred on autophagy adaptors. Indeed, our results identified many of the previously recognized phosphosites, including Ser-152 in the RIPK1 domain and Ser-226 in the TRAF6 binding domain of p62 ${ }^{19}$, but we have identified 
novel residues as well. Importantly, as our understanding of all the adaptors that participate in targeting $M t b$ is incomplete, we noted several autophagy adaptors that were not previously implicated in the response were phosphorylated during infection. Among the cellular networks formed by the phosphorylated proteins during $M t b$ infection, we noted a cluster containing the autophagy receptors p62, optineurin, and TAX1BP1 (Fig. 7A). While p62 was previously shown to target $M t b$ to the autophagosome ${ }^{16}$, the other adaptors with changes in their phosphorylation levels had hitherto not been implicated in autophagy targeting of $M t b$. Autophagy receptor phosphorylation occurred in functional domains of the receptors (Fig. 7B) and changed throughout the time course of $M t b$ infection (Fig. 7C). Of note, the phosphosite we detected in TAX1BP1 is one of the two previously reported serine residues phosphorylated by IKK $\alpha$ in response to $\mathrm{TNF} \alpha$ and $\mathrm{IL}-1$, which results in downregulation of pro-inflammatory $\mathrm{NF}-\kappa \mathrm{B}$ through assembly of the A20 complex ${ }^{37}$. To test the functional consequences of the autophagy receptors phosphorylated during $M t b$ infection, we engineered FLAG-tagged constructs of seven autophagy receptors and transduced RAW macrophages with lentiviral expression vectors for each plasmid. Immunoblotting of transduced macrophages with a FLAG antibody revealed the presence of epitope tagged protein correlating to the predicted protein mobility on SDS-PAGE (Fig. 8A) ${ }^{38}$. We detected unique bands at molecular weight of $92 \mathrm{kDa}$ and $120 \mathrm{kDa}$ consistent with previously published mobilities for immunoblots of TAX1BP1 and NBR1 (Fig. 8B) ${ }^{39,40}$.

To directly assess for recruitment of autophagy receptors to $M t b$ during macrophage infection, we performed immunofluorescence microscopy using antibodies that recognize the FLAG epitope at 6-hours post-infection (Fig. 8C). Consistent with previous results ${ }^{16}, 33 \% \pm 1.7 \%$ (SEM) of mCherry-expressing $M t b$ colocalized with p62. We also detected co-localization of $M t b$ with NBR1 (29\% $\pm 3.3 \%$ co-localization), Optineurin (7\% $\pm 1 \%)$, and TAXBP1 $(26 \% \pm 3.2 \%)$. In contrast, a diffuse staining pattern without $M t b$ colocalization was observed in macrophages expressing BNIP3, BNIP3L, or FUNDC1. Together, these results revealed that p62, Optineurin, and TAX1BP1 are phosphorylated during $M t b$ infection and also colocalize with $M t b$.

\section{TAX1BP1 deficiency results in accumulation of ubiquitylated $M$. tuberculosis}

We used a genetic approach to evaluate the contribution of individual autophagy receptors in the selective autophagy of $M t b$. Previous data showed deficiency of the autophagy receptors p62, NBR1, and NDP52 abrogated targeting of Mtb to autophagosomes ${ }^{15,41}$. In contrast, loss of TAX1BP1 caused an accumulation of ubiquitin-positive Salmonella in a manner requiring its myosin-VI binding domain, implicating a critical role for TAX1BP1 in mediating downstream steps of autophagy targeting. To test the hypothesis that deficiency of autophagy receptors impacts the feedback loop by which autophagy kinases (i.e. phosphorylated TBK1), autophagy adaptors, and ubiquitin assemble around phagocytosed microbes ${ }^{42}$, we performed high throughput immunofluorescence microscopy and automated colocalization analysis to quantify colocalization of these autophagy markers with mCherry-expressing $\mathrm{Mtb}$ in infected $p 62^{-/}$or Taxlbpl $1^{-/}$targeted knockout bone marrow-derived macrophages (Fig. 9A). Consistent with prior results showing that p62 deficient macrophages are impaired in the targeting of Salmonella to ubiquitin $^{43}$, we found decreased targeting of $M t b$ to ubiquitin (FK2) in $p 62^{-/-}$macrophages. In contrast, infected Taxl bp $1^{-/-}$cells displayed increased $M t b$ colocalization with ubiquitin, phospho-TBK1, and p62 (Fig. 9B). Together these findings are consistent with a role for p62 in 
signal amplification of cargo ubiquitylation and TAX1BP1 in targeting intracellular pathogens including Salmonella ${ }^{40}$ and $M t b$ to fully mature autophagosomes.

We next sought to evaluate by an alternative approach whether autophagy components accumulate around $M t b$ in TAX1BP1-deficient macrophages. We generated Cas9-expressing bone-marrow derived macrophages transduced with guide RNAs targeting Tax1bpl 9 or 10. In pooled populations of edited macrophages, editing efficiency at exon 9 or 10 calculated by TIDE analysis $^{44}$ was $90.0 \%$ or $84.8 \%$, respectively. Compared to macrophages transduced with scramble control guides, infected Tax $1 \mathrm{bpl}$ edited macrophages displayed increased $\mathrm{Mtb}$ colocalization with ubiquitin, phospho-TBK1, and p62 (Fig. 10). However, infected Tax1bpl edited or deficient macrophages displayed decreased Mtb colocalization with LC3 (Fig. 11). Thus, TAX1BP1 plays an important role in the clearance of ubiquitin-positive $M t b$, recruitment of p62 and phospho-TBK1, and delivery of $M t b$ to LC3. In summary, our model is that TAX1BP1 is necessary for complete targeting of $M t b$ to the autophagosome in a step downstream from recruitment of p62 and ubiquitin (Fig. 12). 


\section{Discussion}

Despite the global impact of $M t b$ infection on humanity, relatively little is known about the signaling events that occur in the innate immune cells to first encounter this pathogen during infection. Phosphorylation mediated by the serine/threonine kinase, TBK $1^{13}$, and ubiquitylation $^{14,15}$ are two important signaling mechanisms that affect $M t b$ survival, autophagy targeting, and inflammatory responses. However, the substrates phosphorylated or ubiquitinylated during $M t b$ infection were not well described. We therefore set out to comprehensively measure the changes in host protein phosphorylation, ubiquitylation, and abundance, in primary macrophages at five time points following $M t b$ infection. This led to the identification of TAX1BP1, an autophagy receptor not previously known to be involved in $M t b$ infection. With a genetic approach, we found that TAX1BP1 is important for clearance of ubiquitylated $M t b$ and autophagosome maturation. Further work will be necessary to evaluate the impact of TAX1BP1 on $M t b$ growth and inflammatory responses, as well as the functional consequences of the thousands of other substrates that changed in their global abundance or PTM levels during $M t b$ infection.

TAX1BP1 appears to play a distinct role in antibacterial autophagy. Intriguingly, TAX1BP1 is not required for global autophagy in $\mathrm{T}$ cells or MEFs ${ }^{45}$, indicating that TAX1BP1 may play a role in targeting select cargos. However, TAX1BP1 has been implicated as an autophagy adaptor important for clearance of Salmonella in a manner that requires myosin VI, one of TAX1BP1's binding partners ${ }^{40}$. There is also evidence that TAX1BP1 plays a role in the regulation of inflammatory responses as it is critical for the termination of inflammatory responses triggered by NF- $\kappa B$ signaling ${ }^{46}$, and binds to the pro-inflammatory tumor necrosis factor receptor associated factor-6 (TRAF6) ${ }^{47}$ and the anti-inflammatory A20 ubiquitin editing enzyme ${ }^{48}$. Likewise, the fact that it binds to myosin VI indicates that it may play a role in physical movement of large cargo within the cell perhaps to facilitate fusion with the lysosome. Given that TAX1BP1 regulates several different innate immune responses, we aim to determine how TAX1BP1 deficiency ultimately affects in vivo control of $M t b$ infection. 


\section{Methods}

\section{Ethics statement}

An animal use protocol (AUP-2015-11-8096) for mouse use was approved by the Office of Laboratory and Animal Care at the University of California, Berkeley, in adherence with guidelines from the Guide for the Care and Use of Laboratory Animals of the National Institutes of Health.

\section{Mice and Macrophages}

Wild-type C57BL/6J mice obtained from Jackson laboratories. $p 62^{-/}$mice were provided by Herbert Virgin at Washington University, St. Louis, USA. Tax $1 b p 1^{-/}$mice were obtained from Hidekatsu Iha at the University of Oita, Japan. Rosa26-Cas9 knock-in mice ${ }^{49}$ were provided by Gregory Barton at the University of California, Berkeley, USA. Primary murine bone-marrow derived macrophages (BMDMs) were prepared by flushing femurs from 8-12-week-old male and female mice. Bone marrow extracts were differentiated for 7 days and cultured in DMEM-H21 supplemented with 20\% FBS and 15\% MCSF derived from 3T3-MCSF cells. Raw264.7 cells were obtained from the ATCC and cultured in DMEM-H21 supplemented with $10 \%$ FBS and 20 mM HEPES.

\section{Bacterial strain}

M. tuberculosis (Erdman) was used for macrophage infections. M. tuberculosis was grown to log phase in 7H9 liquid media (BD) supplemented with 10\% Middlebrook OADC (Sigma), 0.5\% glycerol, $0.05 \%$ Tween 80 in roller bottles at $37^{\circ} \mathrm{C}$.

\section{Macrophage infection with $M$. tuberculosis}

For proteomics, three independent experiments were performed. BMDMs were seeded at a density of $3 \times 10^{6}$ cells per well in 4 -well cell culture treated dishes. Six dishes were seeded for each experimental condition. The cells were cultured at $37^{\circ} \mathrm{C}$ with $5 \% \mathrm{CO}_{2}$ for 72 -hours prior to infection.

For microscopy to detect FLAG-tagged autophagy receptor colocalization, three coverslips for each experimental condition were seeded with RAW macrophages at a density of $1.2 \mathrm{X} 10^{5}$ cells per coverslip in a 24-well dish. For microscopy to detect ubiquitin, p62, and phospho-TBK1, four wells for each experimental condition were seeded with bone-marrow derived macrophages at a density of $3 \times 10^{4}$ cells per well in a 96-well plate. The cells were cultured for 18-hours prior to infection.

In order to synchronize the start time of the infections, macrophages were infected with $M$. tuberculosis (Erdman strain) using a 'spinfection protocol' at a multiplicity of infection of 10 for proteomics or 2 for microscopy experiments. Logarithmic phase M. tuberculosis suspensions were washed twice with PBS followed by centrifugation for 5 minutes at $183 g$ and sonication to remove and disperse clumps, respectively. M. tuberculosis was resuspended in DMEM with 10\% horse serum. Media was removed from the macrophage monolayers, the bacterial suspension was overlaid, and centrifugation was performed for 10 minutes at $183 g$. For proteomics experiments, cells were returned to macrophage media and incubated until harvesting at 2-, 4-, 6, 8-, or 24-hours post-infection. Uninfected mock controls were harvested at 0-, 6-, and 24-hours 
intervals. For microscopy experiments, infected monolayers were washed twice with PBS and then macrophage media was overlaid. At 8-hours post-infection, the macrophages were washed once with PBS, fixed with $4 \%$ paraformaldehyde, washed twice with PBS, and immunostained.

\section{Cell lysis}

At each time point 0-24-hours post-infection, macrophages were washed with PBS warmed to $37^{\circ} \mathrm{C}$ and $M$. tuberculosis was sterilized on the cell culture plates by the addition of $100 \%$ methanol at $4^{\circ} \mathrm{C}$. The cells were washed twice with PBS and lysed with $6 \mathrm{ml}$ of lysis buffer $(8 \mathrm{M}$ urea, $150 \mathrm{mM} \mathrm{NaCl}, 100 \mathrm{mM}$ ammonium bicarbonate, $\mathrm{pH}$ 8; added per $10 \mathrm{ml}$ of buffer: 1 tablet of Roche mini-complete protease inhibitor EDTA free and 1 tablet of Roche PhosSTOP tablet) prepared fresh before each replicate. Lysates were stored at $-80^{\circ} \mathrm{C}$ until further processing.

\section{Trypsin digest and desalting}

Lysates were sonicated three times with a Sonics VibraCell probe tip sonicator at 7 watts for 10 seconds. In order to remove insoluble precipitate, lysates were centrifuged at $16,100 \mathrm{~g}$ at $4{ }^{\circ} \mathrm{C}$ for 30 minutes. A bicinchoninic acid assay (Pierce) was performed to measure protein concentration in cell lysate supernatants. $10 \mathrm{mg}$ of each clarified lysate was reduced with $4 \mathrm{mM}$ tris(2carboxyethyl)phosphine for 30 minutes at room temperature and alkylated with $2 \mathrm{mM}$ iodoacetamide for 30 minutes at room temperature in the dark. Remaining alkylated agent was quenched with $10 \mathrm{mM}$ 1,4-dithiothreitol for 30 minutes at room temperature in the dark. The samples were diluted with three starting volumes of $100 \mathrm{mM}$ ammonium bicarbonate, $\mathrm{pH}$ 8.0, to reduce the urea concentration. Samples were incubated with $100 \mu \mathrm{g}$ of sequencing grade modified trypsin and incubated at room temperature with rotation for 20 hours. The sample $\mathrm{pH}$ was reduced to approximately 2.0 by the addition of $10 \%$ trifluoroacetic acid (TFA) to a final concentration of $0.3 \%$ trifluoroacetic acid followed by $6 \mathrm{M} \mathrm{HCl}$ at a $1: 100$ volume ratio. Insoluble material was removed by centrifugation at $1989 g$ for 10 minutes.

Peptides were desalted using SepPak C18 solid-phase extraction cartridges (Waters). The columns were activated with $1 \mathrm{ml}$ of $80 \%$ acetonitrile (ACN), $0.1 \%$ TFA, and equilibrated with 3 $\mathrm{ml}$ of $0.1 \%$ TFA. Peptide samples were applied to the columns, and the columns were washed with $3 \mathrm{ml}$ of $0.1 \%$ TFA. Peptides were eluted with $1.1 \mathrm{ml}$ of $40 \%$ ACN, $0.1 \%$ TFA. Peptides were divided for global protein analysis $(10 \mu \mathrm{g})$, phosphoenrichment $(1 \mathrm{mg})$, or diGlyenrichment (remaining sample).

\section{Global protein analysis}

$10 \mu \mathrm{g}$ of peptides were dried with a centrifugal evaporator and stored at $-20^{\circ} \mathrm{C}$ until analysis by liquid chromatograph and mass spectrometry.

\section{Phosphopeptide enrichment by immobilized metal affinity chromatography}

Iron nitriloacetic acid (NTA) resin were prepared in-house by stripping metal ions from nickel nitroloacetic acid agarose resin with $500 \mathrm{mM}$ ethylenediaminetetraacetic acid, $\mathrm{pH} 8.0$ three times. Resin was washed twice with water and $100 \mathrm{mM}$ iron(III) chloride was applied three times. The iron-NTA resin was washed twice with water and once with $0.5 \%$ formic acid. IronNTA beads were resuspended in water to create a $25 \%$ resin slurry. $50 \mu 1$ of Fe-NTA resin slurry was transferred to individual Silica C18 MicroSpin columns (The Nest Group) pre-equilibrated 
with $100 \mu \mathrm{l}$ of $80 \%$ CAN, $0.1 \%$ TFA on a vacuum manifold. Subsequent steps were performed with the Fe-NTA resin loaded on the Silica C18 columns.

Peptide samples were mixed twice with the Fe-NTA resin and allowed to incubate for 2 minutes. The resin was rinsed four times with $200 \mu \mathrm{l}$ of $80 \% \mathrm{ACN}, 0.1 \%$ TFA. In order to equilibrate the chromatography columns, $200 \mu 1$ of $0.5 \%$ formic acid was applied twice to the resin and columns. Peptides were eluted from the resin onto the C18 column by application of $200 \mu 1$ of $500 \mathrm{mM}$ potassium phosphate, $\mathrm{pH}$ 7.0. Peptides were washed twice with $200 \mu 1$ of $0.5 \%$ formic acid. The $\mathrm{C} 18$ columns were removed from the vacuum manifold and eluted twice by centrifugation at $1000 \mathrm{~g}$ with $75 \mu \mathrm{l}$ of $50 \%$ ACN, $0.1 \%$ TFA. Peptides were dried with a centrifugal adaptor and stored at $-20^{\circ} \mathrm{C}$ until analysis by liquid chromatograph and mass spectrometry.

\section{Di-glycine peptide enrichment by immunoprecipitation}

Peptide samples were subjected to ubiquitin remnant immunoaffinity purification with $31 \mu \mathrm{g}$ of ubiquitin remnant antibody (Cell Signaling). Peptides were lyophilized for two days to remove TFA in the elution. The lyophilized peptides were resuspended in $1 \mathrm{ml}$ of IAP buffer (50 mM 4morpholinepropnesulfonic acid, $10 \mathrm{mM}$ disodium hydrogen phosphate, $50 \mathrm{mM}$ sodium chloride, $\mathrm{pH}$ 7.2). Peptides were sonicated and centrifuged for 5 minutes at $16,100 \mathrm{~g}$. Ubiquitin remnant beads were washed twice with IAP buffer and incubated with the peptides at $4^{\circ} \mathrm{C}$ for 90 minutes with rotation. Unbound peptides were separated from the beads after centrifugation at $700 \mathrm{~g}$ for 60 seconds. Beads containing peptides with di-glycine remnants were washed twice with $500 \mu 1$ of water and peptides were eluted twice with $55 \mu 1$ of $0.15 \%$ TFA. Di-glycine remnant peptides were desalted with UltraMicroSpin C18 column (The Nest Group). Desalted peptides were dried with a centrifugal adaptor and stored at $-20^{\circ} \mathrm{C}$ until analysis by liquid chromatograph and mass spectrometry.

\section{Liquid chromatography and mass spectrometry}

Peptides were analyzed in technical duplicate on a ThermoFisher Orbitrap Fusion Lumos Tribid mass spectrometry system equipped with an Easy nLC 1200 ultrahigh-pressure liquid chromatography system interfaced via a Nanospray Flex nanoelectrospray source. Samples were injected on a C18 reverse phase column (25 cm x $75 \mu \mathrm{m}$ packed with ReprosilPur C18 AQ 1.9 um particles). Peptides were separated by an organic gradient from 5 to $30 \%$ ACN in $0.02 \%$ heptafluorobutyric acid over $180 \mathrm{~min}$ at a flow rate of $300 \mathrm{nl} / \mathrm{min}$ for the phosphorylated peptides or unmodified peptides for global abundance. Ubiquitinated peptides were separated by a shorter gradient of $140 \mathrm{~min}$. Spectra were continuously acquired in a data-dependent manner throughout the gradient, acquiring a full scan in the Orbitrap (at 120,000 resolution with an AGC target of 400,000 and a maximum injection time of $50 \mathrm{~ms}$ ) followed by as many MS/MS scans as could be acquired on the most abundant ions in $3 \mathrm{~s}$ in the dual linear ion trap (rapid scan type with an intensity threshold of 5000, HCD collision energy of 32\%, AGC target of 10,000, maximum injection time of $30 \mathrm{~ms}$, and isolation width of $0.7 \mathrm{~m} / \mathrm{z}$ ). Singly and unassigned charge states were rejected. Dynamic exclusion was enabled with a repeat count of 2 , an exclusion duration of $20 \mathrm{~s}$, and an exclusion mass width of $\pm 10 \mathrm{ppm}$. 


\section{Label-free quantitation and statistical analysis}

Mass spectrometry data was assigned to mouse sequences and MS1 intensities were extracted with MaxQuant (version 1.6.0.16) ${ }^{50}$. Data were searched against the SwissProt mouse protein database (downloaded on March 3, 2018). Trypsin (KR/P) was selected allowing for up to two missed cleavages. Variable modification was allowed for $\mathrm{N}$-terminal protein acetylation and methionine oxidation in addition to phosphorylation of serine, threonine, and tyrosine, or diGly modification of lysine, as appropriate. A static modification was assigned to carbamidomethyl cysteine. The other MaxQuant settings were left at the default.

Statistical analysis of MaxQuant-analyzed data was performed with artMS Bioconductor package (version 0.9) which performs the relative quantification using the MSstats Bioconductor package (version 3.14.1) ${ }^{51}$. Contaminants and decoy hits were removed. The samples were normalized across fractions by median-centering the $\log _{2}$-transformed MS1 intensity distributions. The MSstats group comparison function was run with no interaction terms for missing values, no interference, unequal intensity feature variance, restricted technical and biological scope of replication. $\log _{2}$ (fold change) for protein/sites with missing values in one condition but found in $>2$ biological replicates of the other condition of any given comparison were estimated by imputing intensity values from the lowest observed MS1-intensity across samples $^{25} ; p$ values were randomly assigned between 0.05 and 0.01 for illustration purposes. Statistically significant changes were selected by applying a $\log _{2}$-fold-change $(>1.0$ or $<-1.0)$ and an adjusted $p$ value $(<0.05)$ corrected for multiple comparison.

Phosphopeptide data was analyzed with MSstats using the unmodified output data from MaxQuant and phosphosite collapsed data. Phosphopeptide data was collapsed using an in-house script that assigns phosphopeptide sites to the most likely phosphorylated residue in peptides where multiple phosphorylated residues were detected with low phosphorylation probability scores.

\section{Gene enrichment analysis}

Gene enrichment analysis was performed with Metascape (metascape.org) with Custom analysis settings using only GO Biological Processes. Heatmaps were made with the R-based Complex heat map program and the Ward D clustering algorithm.

\section{PhosFate analysis}

Murine phosphorylation sites were mapped to the human proteome using R. Phosphopeptide $\log _{2}$-fold-change profiles were uploaded to the PhosFate Profiler tool (Phosfate.com ${ }^{33}$ ) for kinase and kinase complex predictions.

\section{RNAseq}

Bone marrow-derived macrophages were infected at a MOI of 1 for the 24-hour time point and a MOI of 10 for 2- and 6-hour time points. At the indicated time points, monolayers were washed with PBS, and macrophages were resuspended in $1 \mathrm{ml}$ of TRIzol (ThermoFisher). Following the addition of chloroform $(200 \mu \mathrm{l})$, samples were mixed and centrifuged for $10 \mathrm{~min}$. at $4{ }^{\circ} \mathrm{C}$. An equal volume of $70 \%$ ethanol was added to the aqueous layer, and RNA was extracted using silica spin columns (Invitrogen PureLink RNA Mini Kit). Purified RNA was treated DNAse 
(New England Biolabs) followed by EDTA. RNAseq libraries were prepared from biological triplicate samples by the University of California, Davis, Expression Analysis Core. Differential gene expression analysis was performed by the University of California, Davis, Bioinformatics Core. Transcriptional changes of similar levels between the 24-hour datasets (standard deviation $<1$ for the $\log _{2}$ fold changes) measured in our laboratory and a previously published study of $M t b$ infected bone derived macrophages were included in the analysis.

\section{Cloning and lentiviral transduction of FLAG-tagged autophagy receptors}

PCR products were generated for each autophagy adaptor using the following primer pairs: P30/P2 (p62), P31/P4 (BNIP3), P32/P6 (BNIP3L), P33/P8 (FUNDC1). The remaining autophagy receptors were cloned by splicing with overlap extension PCR with the following primers: P34/P37 and P38/P10 (NBR1), P35/P39 and P40/P12 (Optineurin), P36/P41 and P42/P14 (TAX1BP1). PCR products were cloned into an entry vector encoding a N-terminal triple FLAG tag (pENTR-N-FLAG; Parry et al., in preparation) using sequence- and ligationindependent cloning. The Gateway LR reaction was used to introduce the cloned autophagy receptors into the pLenti CMV Puro DEST destination vector ${ }^{52}$. RAW264.7 cells were transduced and subjected to puromycin selection for 7 days.

\section{Cloning and lentiviral transduction of CRISPR guide RNAs}

Single oligonucleotide guides for three scramble controls and two exons in Tax1bpl selected from the Brie library were closed into lentiGuide puro (Addgene \#52963) using the following primer pairs: P174/P175 (Scramble 1), P176/P177 (Scramble 2), P178/P179 (Scramble 3), P160/P161 (Tax1bpl exon 10), and P164/P165 (Tax1bp1 exon 9). 293T cells were reverse transfected with the vectors encoding scramble or Taxlbpl guides. Bone marrow cells from Rosa26-Cas9 knock-in mice were transduced with retrovirus and differentiated in the presence of puromycin. Transduced and fully-differentiated bone marrow-derived macrophages were cultured for three days in the absence of puromycin before seeding for microscopy experiments.

\section{Western blot}

The anti-FLAG M2 mouse monoclonal antibody was purchased from Sigma-Aldrich. $60 \mu \mathrm{g}$ of protein lysate was separated by SDS-PAGE (BioRad Miniprotean TGX 4-20\%) and transferred onto nitrocellulose membranes. After probing with the anti-FLAG M2 antibody at a dilution of 1:1000, membranes were imaged on an Odyssey scanner (Li-cor).

In order to probe for actin on the same nitrocellulose membrane, antibodies were stripped from the membrane with $0.2 \mathrm{~N}$ sodium hydroxide and re-probed with the $\beta$-actin mouse monoclonal antibody (C4; Santa Cruz Biotechnology, SC-47778).

\section{Immunofluorescence microscopy for FLAG-tagged autophagy receptor colocalization} Infected macrophages were washed with PBS and incubated with blocking and permeabilization buffer $(0.05 \%$ saponin, $5 \%$ fetal calf serum, PBS) for 30 minutes at room temperature. Coverslips were incubated with anti-FLAG M2 mouse monoclonal antibody at a dilution of 1:500 for 3 hours at room temperature and secondary antibody conjugated to fluorophore at a dilution of 1:1000 (Invitrogen). 45 images per coverslip were obtained with a Nikon Eclipse TE2000-E pinning disc confocal microscope equipped with an Andor laser system and Borealis 
beam conditioning unit. Images were obtained with the 100X oil objective for figures and 40X air objective for colocalization.

\section{Immunofluorescence microscopy for p62, ubiquitin, and phospho-TBK1}

Immunostaining for $\mathrm{p} 62$ and ubiquitin was performed with blocking and permeabilization buffer (1\% saponin, $3 \%$ bovine serum albumin) and anti-p62 rabbit monoclonal antibodies (Abcam \#AB109012) at a concentration of $1 \mu \mathrm{g} / \mathrm{ml}$ or anti-ubiquitylated protein antibodies (clone FK2, Millipore Sigma \#04-263) at a dilution of 1:400 for 3 hours at room temperature. Immunostaining for phospho-TBK1 was performed with blocking and permeabilization buffer (0.3\% Triton X-100, 1\% bovine serum albumin in PBS) and anti-phospho-Ser172-TBK1 (D52C2) XP Rabbit monoclonal antibody (Cell Signaling \#5483) for three hours at room temperature. Samples were incubated with a secondary antibody conjugated to Alexa Fluor 488 fluorophore at a dilution of 1:4000 (Invitrogen) and DAPI at a dilution of 1:1000 for one hour at room temperature. 25 images per well were obtained with a Perkin Elmer Opera Phenix High Content Screening confocal microscope at $40 \mathrm{X}$ magnification for colocalization or $63 \mathrm{X}$ for figures. In the images shown from the Rosa26-Cas9 knock-in bone marrow-derived macrophages, low-level background GFP fluorescence was filtered out. Automated colocalization measurements were performed with the Perkin Elmer Harmony software package. A pipeline was created to measure colocalization of $M t b$ and autophagy markers (p62, FK2, and phospho-TBK1) in macrophages infected with two Mtb bacilli.

\section{Immunofluorescence microscopy for LC3}

Immunostaining for LC3 was performed with blocking and permeabilization buffer ( $0.3 \%$ Triton X-100, 2\% bovine serum albumin) and anti-LC3 (clone 2G6, Nanotools \#0260-100/LC3-2G6) at a dilution of 1:200 for 3 hours at room temperature. Samples were incubated with a secondary antibody conjugated to Alexa Fluor 647 fluorophore at a dilution of 1:1000 (ThermoFisher) and DAPI at a dilution of 1:1000 for one hour at room temperature. 39 images per well were obtained with the Opera Phenix confocal microscope at 40X magnification for colocalization or 63X for figures. Automated colocalization measurements were performed in macrophages infected with two $M t b$ bacilli.

\section{Acknowledgements}

We acknowledge members of the Cox and Krogan labs for their comments on this manuscript submission. We thank H. Skip Virgin and Hidekatsu Iha for $p 62^{-/-}$and Tax $1 b p 1^{-/}$mice, respectively. We thank Lori Kohlstaedt at the UC Berkeley proteomics core for assistance in LCMS analysis of our peptide samples, and Christopher Noel for assistance in the development of the Perkin Elmer Harmony microscopy colocalization pipelines. This work was supported by NIH grants P01 AI063302 (N.J.K, J.S.C.), P50 GM082250 (N.J.K), U19 AI106754 (N.J.K), DP1 AI124619 (J.S.C), and R01 AI120694 (N.J.K and J.S.C). J.M.B was supported by T32 training grant (4T32HL007185-39 \& -40, Dean Sheppard PI) and NIH K12 (5K12HL119997-05, David Erle PI). Research reported in this publication was supported by the Office of The Director, National Institutes of Health of the National Institutes of Health under Award Number S10OD021828 for the Opera Phenix and associated staff support. The content is solely the 
bioRxiv preprint doi: https://doi.org/10.1101/534917; this version posted January 31, 2019. The copyright holder for this preprint (which was not certified by peer review) is the author/funder. All rights reserved. No reuse allowed without permission.

responsibility of the authors and does not necessarily represent the official views of the National Institutes of Health. 


\section{Figure Legends}

Fig. 1. Proteomics and RNAseq profiling of $M t b$ infected bone marrow-derived macrophages. (A) Schematic for the experimental design indicating the hours post-infection at which point mock-infected or Mtb infected macrophages were harvested and peptides generated for global protein abundance measurements by LC-MS. Phosphorylated peptides (Ph) or ubiquitylated peptides containing the diGly-remnant (GG) were separately enriched. (B) Replica plots of MS intensity measurements for individual peptides in global protein abundance $(A B)$, phosphopeptide (PH), or ubiquitylated peptide (UB) samples. The correlation coefficient is displayed (R). (C) Percent of total changes occurring at each time point 2-24-hours post-infection for global protein abundance, phosphorylation, or ubiquitylation. $(D)$ Correlation plot of changes in gene transcription and protein abundance $(\mathrm{AB})$, phosphorylation $(\mathrm{PH})$, or ubiquitylation (UB) at 24-hours post-infection with $M t b$. Proteins with no statistically significant changes during $M t b$ infection are colored black. Non-imputed proteins with statistically significant changes during $M t b$ infection are colored blue. Imputed proteins during $M t b$ infection are colored red. The correlation coefficient for non-imputed and imputed proteins are both shown.

Fig. 2. Comparison of macrophage proteins changing in abundance, phosphorylation, or ubiquitylation during $M t b$ infection. (A) Volcano plots displaying proteins changing in abundance, phosphorylation, or ubiquitylation at 24-hours post-infection. Proteins with a $\log _{2}$ (fold change) greater than 1 are colored red. Proteins with a $\log _{2}$ (fold change) less than -1 are colored blue. Proteins with a $p$ value less than 0.05 (or $-\log 10$ ( $p$ value) greater than 1.3) are above the horizontal black line. Phosphorylated residues and ubiquitylated lysine residues are noted. $(B)$ Venn diagram displaying the number of unique and overlapping proteins changing in abundance $(\mathrm{AB})$, phosphorylation $(\mathrm{PH})$, or ubiquitylation (UB) in the aggregate measurements 224-hours post-infection. $(C)$ Circos visualization of protein and functional enrichment term overlap in the aggregate measurements 2-24-hours post-infection. Identical protein pairs are linked by a blue line. Protein pairs falling into the same enriched term are linked by a purple line. (D) Enriched ontogeny clusters highlighting commonly enriched pathways. Pathways are colored coded into common groups (trafficking, metabolism, signal transduction, immunity).

Fig. 3. Host protein abundance changes during infection with $M t b$. (A) Cluster analysis and heat map displaying the $\log _{2}$ (fold change) in protein abundance for non-imputed and imputed proteins with a $p$ value less than 0.05 . The most statistically significant functional enrichment term and representative proteins are indicated for each class of proteins. (B) Graph of the functional enriched terms for protein class 1 and 2.

Fig. 4. Changes in host protein ubiquitylation during Mtb infection. (A) Cluster analysis and heat map displaying the $\log _{2}$ (fold change) in ubiquitylated peptide levels for non-imputed and imputed proteins with a $p$ value less than 0.05 . The most statistically significant functional enrichment term and representative proteins are indicated for each class of proteins. $(B)$ Heat map visualization of functional enriched terms for each protein class. 
Fig. 5. Changes in host protein phosphorylation during Mtb infection. (A) Cluster analysis and heat map displaying the $\log _{2}$ (fold change) in phosphorylated peptide levels for non-imputed and imputed proteins with a $p$ value less than 0.05 . The most statistically significant functional enrichment term and representative proteins are indicated for each class of proteins. $(B)$ Heat map visualization of functional enriched terms for each protein class.

Fig. 6. Protein kinase and effector complex activity predictions. (A) Cluster analysis and heat map displaying the predicted host protein kinase activity. Mouse phosphopeptide sites were mapped to the human proteome and kinase activity predictions were performed with PhosFate ${ }^{33}$. (B). Effector complex activity predictions. Effector complexes containing ubiquitin ligases (bold) are listed.

Fig. 7. Autophagy receptor phosphorylation during $M t b$ infection. (A) Table showing the number of unique phosphosites in each autophagy receptor at 2-24-hours post-infection. * $p$ value less than 0.05 and $\log _{2}$ (fold change) greater than 1 or less than -1 for at least one of the phosphosites.

$(B)$ Domain organization displaying post-translational modifications in autophagy receptors. The LC3-interacting region (LIR), ubiquitin binding domain (UBAN, UBZ), myosin-6 binding domain (MYO6), SKIP carboxyl homology domain (SKICH), and TANK-binding kinase-1 (TBK1) binding domains are labeled. $(C-G)$ Volcano plots highlighting changes in autophagy receptor phosphorylation at each time point 2-24-hours post- $M t b$ infection. Proteins with a $\log _{2}$ (fold change) greater than 1 are colored red. Proteins with a $\log _{2}$ (fold change) less than -1 are colored blue. Proteins with a $p$ value less than 0.05 (or $-\log 10$ ( $p$ value) greater than 1.3) are above the horizontal black line. Phosphorylated residues and ubiquitylated lysine residues are noted.

Fig. 8. TAX1BP1, p62, optineurin, and NBR1 colocalize with $M t b$. (A and $B$ ) Immunoblot showing expression of FLAG-tagged autophagy receptors in RAW macrophages. RAW macrophages were transduced with FLAG-tagged autophagy receptor constructs and cell lysates probed with $\alpha$-FLAG and $\alpha$-actin antisera. Immunoblots were imaged with a short exposure time $(A)$ or a long exposure time $(B)$. $(C)$ Fluorescence images of RAW macrophages expressing FLAG-tagged autophagy receptors (green) with mCherry-expressing $M t b$ (red).

Fig. 9. Accumulation of $M t b$ targeted to ubiquitin (FK2), p62, and phospho-TBK1 in TAX1BP1 targeted knockout macrophages. (A) Fluorescence images of wild-type, $p 62^{-/-}$, or Tax1bp1 $1^{-/-}$bone marrow-derived macrophages infected with mCherry-expressing $M t b$ (red) and immunostained with antibodies to ubiquitin (FK2), p62, or phospho-TBK1 (green). (B) Quantitative analysis of $M t b$ colocalization with ubiquitin, p62, and phospho-TBK1 and 8-hours post-infection. Results are the means \pm SEM for five technical replicates and representative of three independent experiments. $* * * p$ value less than 0.001 by $t$-test. ns (non-significant). 
Fig. 10. Accumulation of $M t b$ targeted to ubiquitin (FK2), p62, and phospho-TBK1 in TAX1BP1 edited macrophages. (A) Fluorescence images of bone marrow-derived macrophages transduced with scramble control guide RNAs or Taxlbpl exon 10 or 9 guide RNAs infected with mCherry-expressing $M t b$ (red) and immunostained with antibodies to ubiquitin (FK2), p62, or phospho-TBK1 (green). Nuclei were stained with DAPI (blue). (B) Quantitative analysis of $M t b$ colocalization with ubiquitin, p62, and phospho-TBK1 at 8-hours post-infection. Results are the means \pm SEM for four technical replicates. ${ }^{*} p$ value less than 0.05 by $t$-test. $* * p$ value less than 0.01 . *** $p$ value less than 0.001 .

Fig. 11. TAX1BP1 deficiency abrogates targeting of Mtb to LC3. (A) Fluorescence images of bone-marrow derived macrophages infected with mCherry-expressing $M t b$ (red) and immunostained with $\alpha$-LC3 antisera (green). Nuclei were stained with DAPI (blue). (B) Quantitative analysis of $M t b$ colocalization with LC3 at 8-hours post-infection. Results are the means $\pm \mathrm{SEM}$ for four technical replicates and representative of two independent experiments. * $p$ value less than 0.05 by $t$-test. ** $p$ value less than 0.01 . *** $p$ value less than 0.001 .

Fig 12. A model showing the role of p62 in amplification of ubiquitin recruitment to $M t b$ and TAX1BP1 in completing the targeting of $M t b$ to selective autophagy in a step downstream of p62 recruitment.

Fig. S1. Unique host proteins identified at each time point 2-24-hours post-infection with $M t b$ or mock infection. $(A-C)$ Graph of the number of unique proteins identified by mass spectrometry in biological triplicate, technical duplicate samples for each experimental condition from unenriched $(A)$, phosphoenriched $(B)$, or di-Gly enriched $(C)$ peptide samples.

Fig. S2. Host protein abundance changes during a time course of Mtb infection. Volcano plots displaying the proteins changing in abundance during $M t b$ infection. Proteins with a $p$ value less than 0.05 and $\log _{2}$ (fold change) greater than 1 are colored red. Proteins with a $p$ value less than 0.05 and $\log _{2}$ (fold change) less than -1 are colored blue.

Fig. S3. Changes in host protein phosphorylation during a time course of $M t b$ infection. Volcano plots displaying the changes in phosphopeptides during $M t b$ infection. Phosphopeptides with a $p$ value less than 0.05 and $\log _{2}$ (fold change) greater than 1 are colored red. Phosphopeptides with a $p$ value less than 0.05 and $\log _{2}$ (fold change) less than -1 are colored blue.

Fig. S4. Changes in host protein ubiquitylation during a time course of $M t b$ infection. Volcano plots displaying the changes in di-Gly modified peptides during Mtb infection. Ubiquitylated peptides with a $p$ value less than 0.05 and $\log _{2}$ (fold change) greater than 1 are colored red. Ubiquitylated peptides with a $p$ value less than 0.05 and $\log _{2}$ (fold change) less than -1 are colored blue.

Fig. S5. Correlation between host transcript and protein abundance changes during $M t b$ infection. $(A, B)$ Correlation plot of changes in gene transcription and protein abundance 2- $(A)$ and 6-hours $(B)$ post-infection with $M t b$. Proteins with no statistically significant changes during $M t b$ infection are colored black. Non-imputed proteins with statistically significant changes 
during $M t b$ infection are colored blue. Imputed proteins during $M t b$ infection are colored red. The correlation coefficient for non-imputed and imputed proteins are both shown.

Fig. S6. Functional enrichment terms of host proteins changes during $M t b$ infection. (A-C) Heat map displaying functional enrichment terms for the classes of protein changes (Fig. 3-5) during $M t b$ infection. Non-imputed (NI) and imputed (IMP) measurements were separated for the analysis.

Table S1. R values for peptide intensity levels from biological and technical replicates.

Table S2. Primers used in this study.

Table S3. Autophagy receptors changing in protein abundance, phosphorylation, or ubiquitylation during $M t b$ infection.

Table S4. Protein abundance changes during Mtb infection.

Table S5. Changes in phosphorylation during $M t b$ infection.

Table S6. Changes in ubiquitylation during $M t b$ infection. 


\section{References}

1. World Health Organization. Global tuberculosis report 2018. 2018: Accessed online: November 29, 2018.

2. Dheda K, Gumbo T, Maartens G, Dooley KE, McNerney R, Murray M, Furin J, Nardell EA, London L, Lessem E, Theron G, van Helden P, Niemann S, Merker M, Dowdy D, Van Rie A, Siu GK, Pasipanodya JG, Rodrigues C, Clark TG, Sirgel FA, Esmail A, Lin HH, Atre SR, Schaaf HS, Chang KC, Lange C, Nahid P, Udwadia ZF, Horsburgh CR,Jr, Churchyard GJ, Menzies D, Hesseling AC, Nuermberger E, McIlleron H, Fennelly KP, Goemaere E, Jaramillo E, Low M, Jara CM, Padayatchi N, Warren RM. The epidemiology, pathogenesis, transmission, diagnosis, and management of multidrug-resistant, extensively drug-resistant, and incurable tuberculosis. Lancet Respir Med. 2017 Mar 15 PMID: 28344011.

3. Dowdy DW, Theron G, Tornheim JA, Kendall EA. Drug-resistant tuberculosis in 2017: At a crossroads. Lancet Respir Med. 2017 Mar 15 PMID: 28344006 PMCID: PMC6003863.

4. Leisching G, Pietersen RD, van Heerden C, van Helden P, Wiid I, Baker B. RNAseq reveals hypervirulence-specific host responses to M. tuberculosis infection. Virulence. 2017 Aug 18; 8(6): 848-858. PMID: 27763806 PMCID: PMC5626229.

5. Braverman J, Sogi KM, Benjamin D, Nomura DK, Stanley SA. HIF-1alpha is an essential mediator of IFN-gamma-dependent immunity to mycobacterium tuberculosis. J Immunol. 2016 Aug 15; 197(4): 1287-1297. PMID: 27430718 PMCID: PMC4976004.

6. Sasindran SJ, Torrelles JB. Mycobacterium tuberculosis infection and inflammation: What is beneficial for the host and for the bacterium? Front Microbiol. 2011 Jan 26; 2: 2. PMID: 21687401 PMCID: PMC3109289.

7. Kornfeld H, Mancino G, Colizzi V. The role of macrophage cell death in tuberculosis. Cell Death Differ. 1999 Jan; 6(1): 71-78. PMID: 10200550.

8. Xu S, Cooper A, Sturgill-Koszycki S, van Heyningen T, Chatterjee D, Orme I, Allen P, Russell DG. Intracellular trafficking in mycobacterium tuberculosis and mycobacterium aviuminfected macrophages. J Immunol. 1994 Sep 15; 153(6): 2568-2578. PMID: 8077667.

9. Zhang N, Kima PE. Leishmania infection engages non-receptor protein kinases differentially to persist in infected hosts. Front Immunol. 2016 Apr 18; 7: 146. PMID: 27148265 PMCID: PMC4834468.

10. Huang J, Brumell JH. Bacteria-autophagy interplay: A battle for survival. Nat Rev Microbiol. 2014 Feb; 12(2): 101-114. PMID: 24384599.

11. Choy A, Dancourt J, Mugo B, O'Connor TJ, Isberg RR, Melia TJ, Roy CR. The legionella effector RavZ inhibits host autophagy through irreversible Atg8 deconjugation. Science. 2012 Nov 23; 338(6110): 1072-1076. PMID: 23112293 PMCID: PMC3682818. 
12. Liu S, Cai X, Wu J, Cong Q, Chen X, Li T, Du F, Ren J, Wu YT, Grishin NV, Chen ZJ. Phosphorylation of innate immune adaptor proteins MAVS, STING, and TRIF induces IRF3 activation. Science. 2015 Mar 13; 347(6227): aaa2630. PMID: 25636800.

13. Stanley SA, Raghavan S, Hwang WW, Cox JS. Acute infection and macrophage subversion by mycobacterium tuberculosis require a specialized secretion system. Proc Natl Acad Sci U S A. 2003 Oct 28; 100(22): 13001-13006. PMID: 14557536 PMCID: PMC240734.

14. Manzanillo PS, Ayres JS, Watson RO, Collins AC, Souza G, Rae CS, Schneider DS, Nakamura K, Shiloh MU, Cox JS. The ubiquitin ligase parkin mediates resistance to intracellular pathogens. Nature. 2013 Sep 26; 501(7468): 512-516. PMID: 24005326.

15. Franco LH, Nair VR, Scharn CR, Xavier RJ, Torrealba JR, Shiloh MU, Levine B. The ubiquitin ligase Smurf1 functions in selective autophagy of mycobacterium tuberculosis and anti-tuberculous host defense. Cell Host Microbe. 2017 Sep 13; 22(3): 421-423. PMID: 28910640 PMCID: PMC5818210.

16. Watson RO, Manzanillo PS, Cox JS. Extracellular M. tuberculosis DNA targets bacteria for autophagy by activating the host DNA-sensing pathway. Cell. 2012 Aug 17; 150(4): 803-815. PMID: 22901810 PMCID: PMC3708656.

17. Manzanillo PS, Shiloh MU, Portnoy DA, Cox JS. Mycobacterium tuberculosis activates the DNA-dependent cytosolic surveillance pathway within macrophages. Cell Host Microbe. 2012 May 17; 11(5): 469-480. PMID: 22607800 PMCID: PMC3662372.

18. Levine B, Mizushima N, Virgin HW. Autophagy in immunity and inflammation. Nature. 2011 Jan 20; 469(7330): 323-335. PMID: 21248839 PMCID: PMC3131688.

19. Richter B, Sliter DA, Herhaus L, Stolz A, Wang C, Beli P, Zaffagnini G, Wild P, Martens S, Wagner SA, Youle RJ, Dikic I. Phosphorylation of OPTN by TBK1 enhances its binding to ub chains and promotes selective autophagy of damaged mitochondria. Proc Natl Acad Sci U S A. 2016 Apr 12; 113(15): 4039-4044. PMID: 27035970 PMCID: PMC4839414.

20. Peng H, Yang J, Li G, You Q, Han W, Li T, Gao D, Xie X, Lee BH, Du J, Hou J, Zhang T, Rao H, Huang Y, Li Q, Zeng R, Hui L, Wang H, Xia Q, Zhang X, He Y, Komatsu M, Dikic I, Finley D, Hu R. Ubiquitylation of p62/sequestosome1 activates its autophagy receptor function and controls selective autophagy upon ubiquitin stress. Cell Res. 2017 May; 27(5): 657-674. PMID: 28322253 PMCID: PMC5520855.

21. Penn BH, Netter Z, Johnson JR, Von Dollen J, Jang GM, Johnson T, Ohol YM, Maher C, Bell SL, Geiger K, Golovkine G, Du X, Choi A, Parry T, Mohapatra BC, Storck MD, Band H, Chen C, Jager S, Shales M, Portnoy DA, Hernandez R, Coscoy L, Cox JS, Krogan NJ. An mtbhuman protein-protein interaction map identifies a switch between host antiviral and antibacterial responses. Mol Cell. 2018 Aug 16; 71(4): 637-648.e5. PMID: 30118682. 
22. Sogi KM, Lien KA, Johnson JR, Krogan NJ, Stanley SA. The tyrosine kinase inhibitor gefitinib restricts mycobacterium tuberculosis growth through increased lysosomal biogenesis and modulation of cytokine signaling. ACS Infect Dis. 2017 Jun 5 PMID: 28537707.

23. Udeshi ND, Mani DR, Eisenhaure T, Mertins P, Jaffe JD, Clauser KR, Hacohen N, Carr SA. Methods for quantification of in vivo changes in protein ubiquitination following proteasome and deubiquitinase inhibition. Mol Cell Proteomics. 2012 May; 11(5): 148-159. PMID: 22505724 PMCID: PMC3418844.

24. Thingholm TE, Larsen MR. Phosphopeptide enrichment by immobilized metal affinity chromatography. Methods Mol Biol. 2016; 1355: 123-133. PMID: 26584922.

25. Webb-Robertson BJ, Wiberg HK, Matzke MM, Brown JN, Wang J, McDermott JE, Smith RD, Rodland KD, Metz TO, Pounds JG, Waters KM. Review, evaluation, and discussion of the challenges of missing value imputation for mass spectrometry-based label-free global proteomics. J Proteome Res. 2015 May 1; 14(5): 1993-2001. PMID: 25855118 PMCID: PMC4776766.

26. Edfors F, Danielsson F, Hallstrom BM, Kall L, Lundberg E, Ponten F, Forsstrom B, Uhlen M. Gene-specific correlation of RNA and protein levels in human cells and tissues. Mol Syst Biol. 2016 Oct 20; 12(10): 883. PMID: 27951527 PMCID: PMC5081484.

27. Liu Y, Beyer A, Aebersold R. On the dependency of cellular protein levels on mRNA abundance. Cell. 2016 Apr 21; 165(3): 535-550. PMID: 27104977.

28. Ashburner M, Ball CA, Blake JA, Botstein D, Butler H, Cherry JM, Davis AP, Dolinski K, Dwight SS, Eppig JT, Harris MA, Hill DP, Issel-Tarver L, Kasarskis A, Lewis S, Matese JC, Richardson JE, Ringwald M, Rubin GM, Sherlock G. Gene ontology: Tool for the unification of biology. the gene ontology consortium. Nat Genet. 2000 May; 25(1): 25-29. PMID: 10802651 PMCID: PMC3037419.

29. Tripathi S, Pohl MO, Zhou Y, Rodriguez-Frandsen A, Wang G, Stein DA, Moulton HM, DeJesus P, Che J, Mulder LC, Yanguez E, Andenmatten D, Pache L, Manicassamy B, Albrecht RA, Gonzalez MG, Nguyen Q, Brass A, Elledge S, White M, Shapira S, Hacohen N, Karlas A, Meyer TF, Shales M, Gatorano A, Johnson JR, Jang G, Johnson T, Verschueren E, Sanders D, Krogan N, Shaw M, Konig R, Stertz S, Garcia-Sastre A, Chanda SK. Meta- and orthogonal integration of influenza "OMICs" data defines a role for UBR4 in virus budding. Cell Host Microbe. 2015 Dec 9; 18(6): 723-735. PMID: 26651948 PMCID: PMC4829074.

30. Moreira-Teixeira L, Mayer-Barber K, Sher A, O'Garra A. Type I interferons in tuberculosis: Foe and occasionally friend. J Exp Med. 2018 May 7; 215(5): 1273-1285. PMID: 29666166 PMCID: PMC5940272.

31. Fu YR, Gao KS, Ji R, Yi ZJ. Differential transcriptional response in macrophages infected with cell wall deficient versus normal mycobacterium tuberculosis. Int J Biol Sci. 2015 Jan 1; 11(1): 22-30. PMID: 25552926 PMCID: PMC4278251. 
32. Ranjbar S, Jasenosky LD, Chow N, Goldfeld AE. Regulation of mycobacterium tuberculosisdependent HIV-1 transcription reveals a new role for NFAT5 in the toll-like receptor pathway. PLoS Pathog. 2012; 8(4): e1002620. PMID: 22496647 PMCID: PMC3320587.

33. Ochoa D, Jonikas M, Lawrence RT, El Debs B, Selkrig J, Typas A, Villen J, Santos SD, Beltrao P. An atlas of human kinase regulation. Mol Syst Biol. 2016 Dec 1; 12(12): 888. PMID: 27909043 PMCID: PMC5199121.

34. Lapek JD,Jr, Lewinski MK, Wozniak JM, Guatelli J, Gonzalez DJ. Quantitative temporal viromics of an inducible HIV-1 model yields insight to global host targets and phosphodynamics associated with protein vpr. Mol Cell Proteomics. 2017 Aug; 16(8): 1447-1461. PMID: 28606917 PMCID: PMC5546197.

35. Israel A. The IKK complex, a central regulator of NF-kappaB activation. Cold Spring Harb Perspect Biol. 2010 Mar; 2(3): a000158. PMID: 20300203 PMCID: PMC2829958.

36. Rahman A, Sobia P, Gupta N, Kaer LV, Das G. Mycobacterium tuberculosis subverts the TLR-2-MyD88 pathway to facilitate its translocation into the cytosol. PLoS One. 2014 Jan 27; 9(1): e86886. PMID: 24475192 PMCID: PMC3903598.

37. Shembade N, Pujari R, Harhaj NS, Abbott DW, Harhaj EW. The kinase IKKalpha inhibits activation of the transcription factor NF-kappaB by phosphorylating the regulatory molecule TAX1BP1. Nat Immunol. 2011 Jul 17; 12(9): 834-843. PMID: 21765415 PMCID: PMC3205447.

38. Shang P, Valapala M, Grebe R, Hose S, Ghosh S, Bhutto IA, Handa JT, Lutty GA, Lu L, Wan J, Qian J, Sergeev Y, Puertollano R, Zigler JS,Jr, Xu GT, Sinha D. The amino acid transporter SLC36A4 regulates the amino acid pool in retinal pigmented epithelial cells and mediates the mechanistic target of rapamycin, complex 1 signaling. Aging Cell. 2017 Apr; 16(2): 349-359. PMID: 28083894 PMCID: PMC5334531.

39. Ito S, Tanaka Y, Oshino R, Aiba K, Thanasegaran S, Nishio N, Isobe K. GADD34 inhibits activation-induced apoptosis of macrophages through enhancement of autophagy. Sci Rep. 2015 Feb 9; 5: 8327. PMID: 25659802 PMCID: PMC4321179.

40. Tumbarello DA, Manna PT, Allen M, Bycroft M, Arden SD, Kendrick-Jones J, Buss F. The autophagy receptor TAX1BP1 and the molecular motor myosin VI are required for clearance of salmonella typhimurium by autophagy. PLoS Pathog. 2015 Oct 9; 11(10): e1005174. PMID: 26451915 PMCID: PMC4599966.

41. Watson RO, Bell SL, MacDuff DA, Kimmey JM, Diner EJ, Olivas J, Vance RE, Stallings CL, Virgin HW, Cox JS. The cytosolic sensor cGAS detects mycobacterium tuberculosis DNA to induce type I interferons and activate autophagy. Cell Host Microbe. 2015 Jun 10; 17(6): 811819. PMID: 26048136 PMCID: PMC4466081. 
42. Pilli M, Arko-Mensah J, Ponpuak M, Roberts E, Master S, Mandell MA, Dupont N, Ornatowski W, Jiang S, Bradfute SB, Bruun JA, Hansen TE, Johansen T, Deretic V. TBK-1 promotes autophagy-mediated antimicrobial defense by controlling autophagosome maturation. Immunity. 2012 Aug 24; 37(2): 223-234. PMID: 22921120 PMCID: PMC3428731.

43. Zheng YT, Shahnazari S, Brech A, Lamark T, Johansen T, Brumell JH. The adaptor protein p62/SQSTM1 targets invading bacteria to the autophagy pathway. J Immunol. 2009 Nov 1; 183(9): 5909-5916. PMID: 19812211.

44. Brinkman EK, Chen T, Amendola M, van Steensel B. Easy quantitative assessment of genome editing by sequence trace decomposition. Nucleic Acids Res. 2014 Dec 16; 42(22): e168. PMID: 25300484 PMCID: PMC4267669.

45. Whang MI, Tavares RM, Benjamin DI, Kattah MG, Advincula R, Nomura DK, Debnath J, Malynn BA, Ma A. The ubiquitin binding protein TAX1BP1 mediates autophagasome induction and the metabolic transition of activated T cells. Immunity. 2017 Mar 21; 46(3): 405-420. PMID: 28314591 PMCID: PMC5400745.

46. Shembade N, Harhaj NS, Liebl DJ, Harhaj EW. Essential role for TAX1BP1 in the termination of TNF-alpha-, IL-1- and LPS-mediated NF-kappaB and JNK signaling. EMBO J. 2007 Sep 5; 26(17): 3910-3922. PMID: 17703191 PMCID: PMC1994124.

47. Ling L, Goeddel DV. T6BP, a TRAF6-interacting protein involved in IL-1 signaling. Proc Natl Acad Sci U S A. 2000 Aug 15; 97(17): 9567-9572. PMID: 10920205 PMCID: PMC16905.

48. Parvatiyar K, Barber GN, Harhaj EW. TAX1BP1 and A20 inhibit antiviral signaling by targeting TBK1-IKKi kinases. J Biol Chem. 2010 May 14; 285(20): 14999-15009. PMID: 20304918 PMCID: PMC2865285.

49. Platt RJ, Chen S, Zhou Y, Yim MJ, Swiech L, Kempton HR, Dahlman JE, Parnas O, Eisenhaure TM, Jovanovic M, Graham DB, Jhunjhunwala S, Heidenreich M, Xavier RJ, Langer R, Anderson DG, Hacohen N, Regev A, Feng G, Sharp PA, Zhang F. CRISPR-Cas9 knockin mice for genome editing and cancer modeling. Cell. 2014 Oct 9; 159(2): 440-455. PMID: 25263330 PMCID: PMC4265475.

50. Cox J, Mann M. MaxQuant enables high peptide identification rates, individualized p.p.b.range mass accuracies and proteome-wide protein quantification. Nat Biotechnol. 2008 Dec; 26(12): 1367-1372. PMID: 19029910.

51. Choi M, Chang CY, Clough T, Broudy D, Killeen T, MacLean B, Vitek O. MSstats: An R package for statistical analysis of quantitative mass spectrometry-based proteomic experiments. Bioinformatics. 2014 Sep 1; 30(17): 2524-2526. PMID: 24794931.

52. Campeau E, Ruhl VE, Rodier F, Smith CL, Rahmberg BL, Fuss JO, Campisi J, Yaswen P, Cooper PK, Kaufman PD. A versatile viral system for expression and depletion of proteins in 
bioRxiv preprint doi: https://doi.org/10.1101/534917; this version posted January 31, 2019. The copyright holder for this preprint (which was not certified by peer review) is the author/funder. All rights reserved. No reuse allowed without permission.

mammalian cells. PLoS One. 2009 Aug 6; 4(8): e6529. PMID: 19657394 PMCID: PMC2717805. 
Fig. S1

A

\section{Protein Abundance}

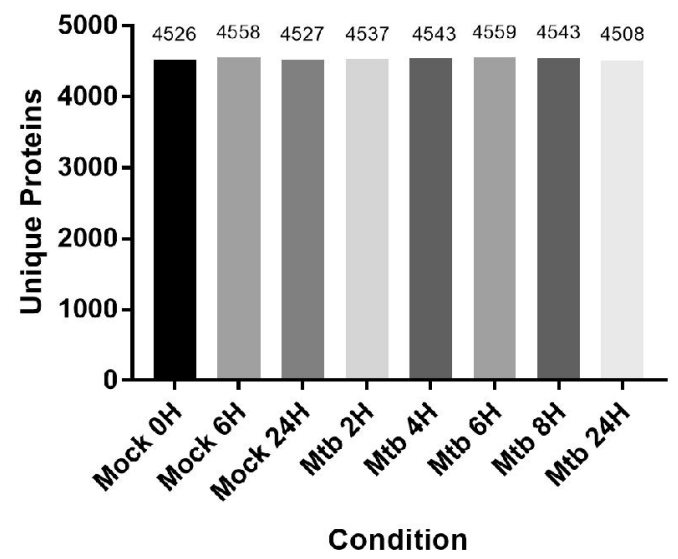

\section{Phosphorylated Proteins}

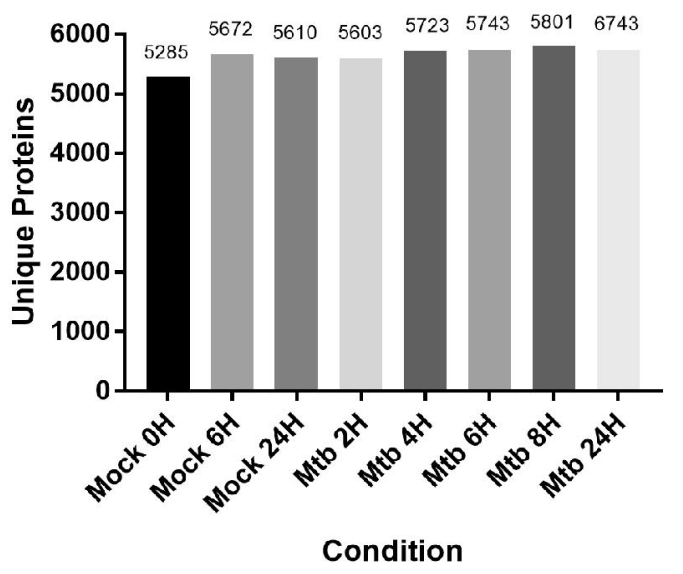

Ubiquitylated Proteins

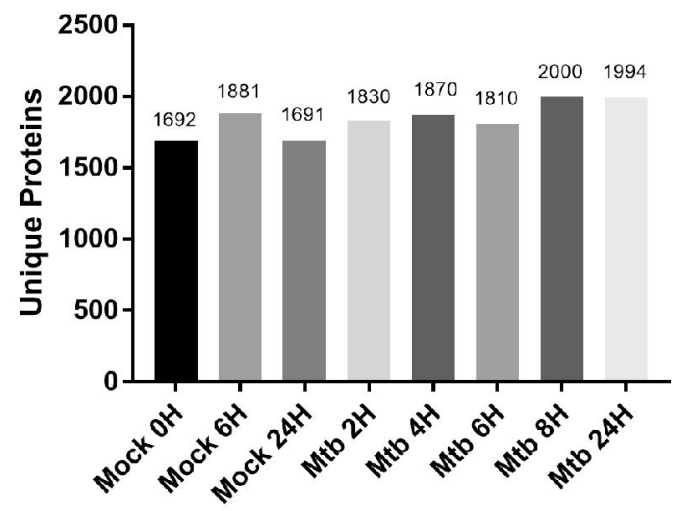

Condition 
Fig. S2

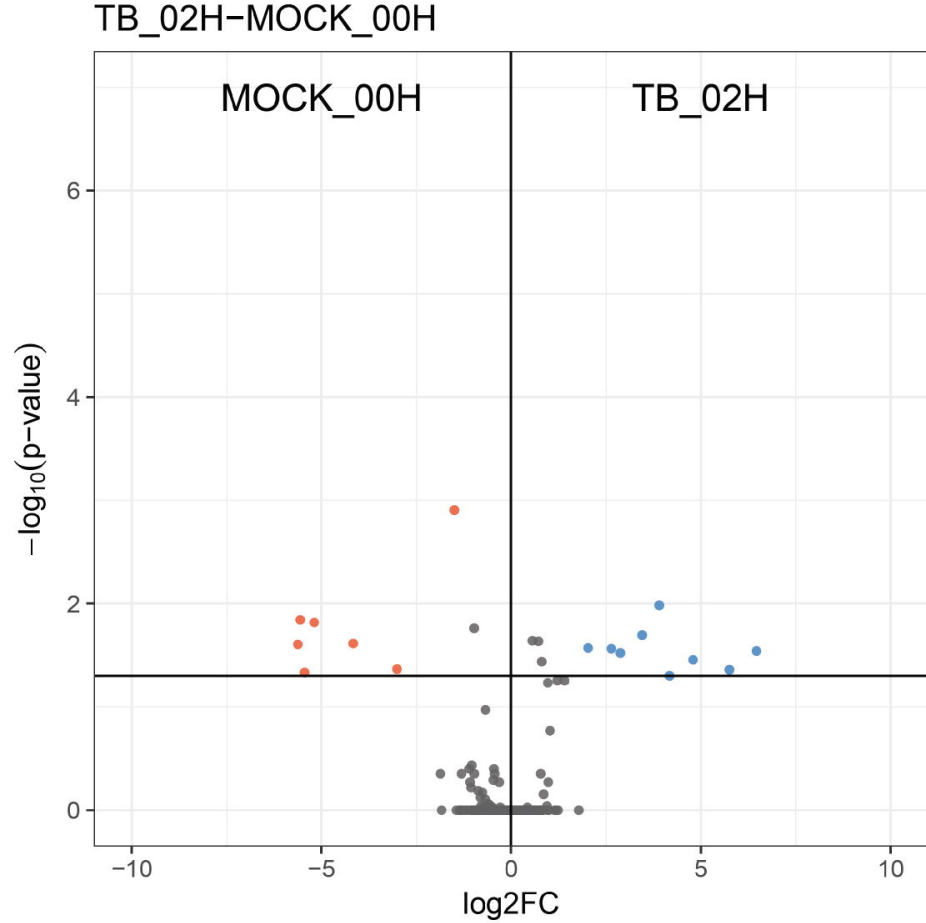

TB_08H-MOCK_06H

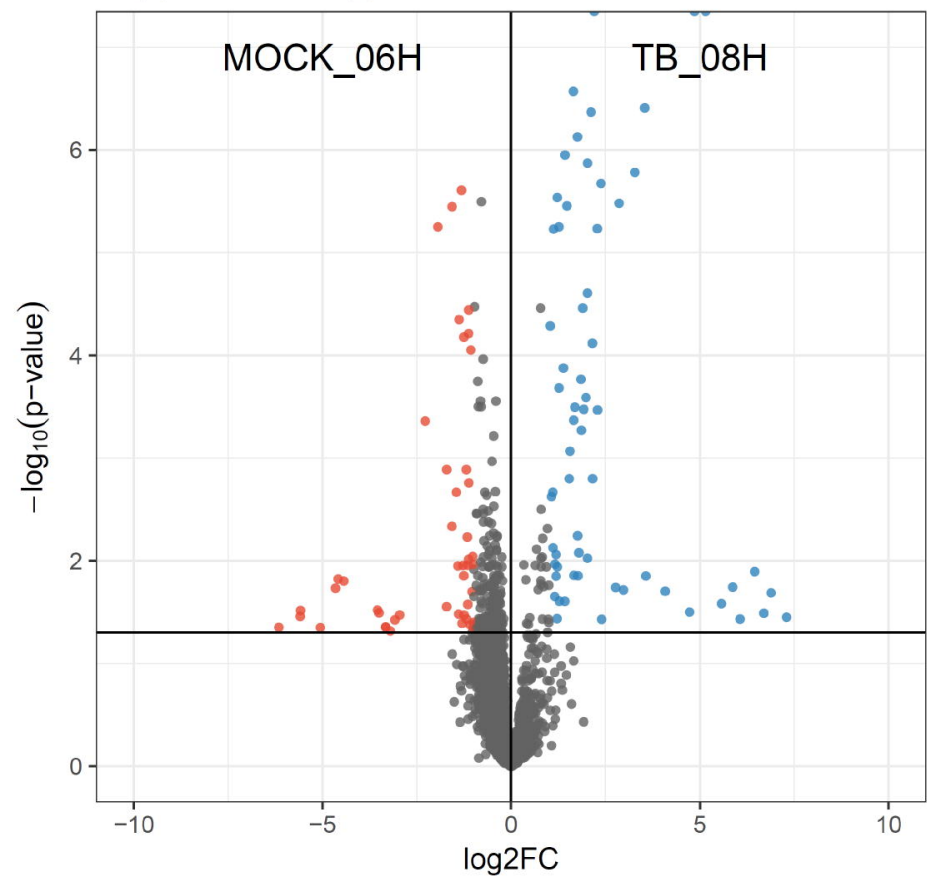

TB_04H-MOCK_OOH

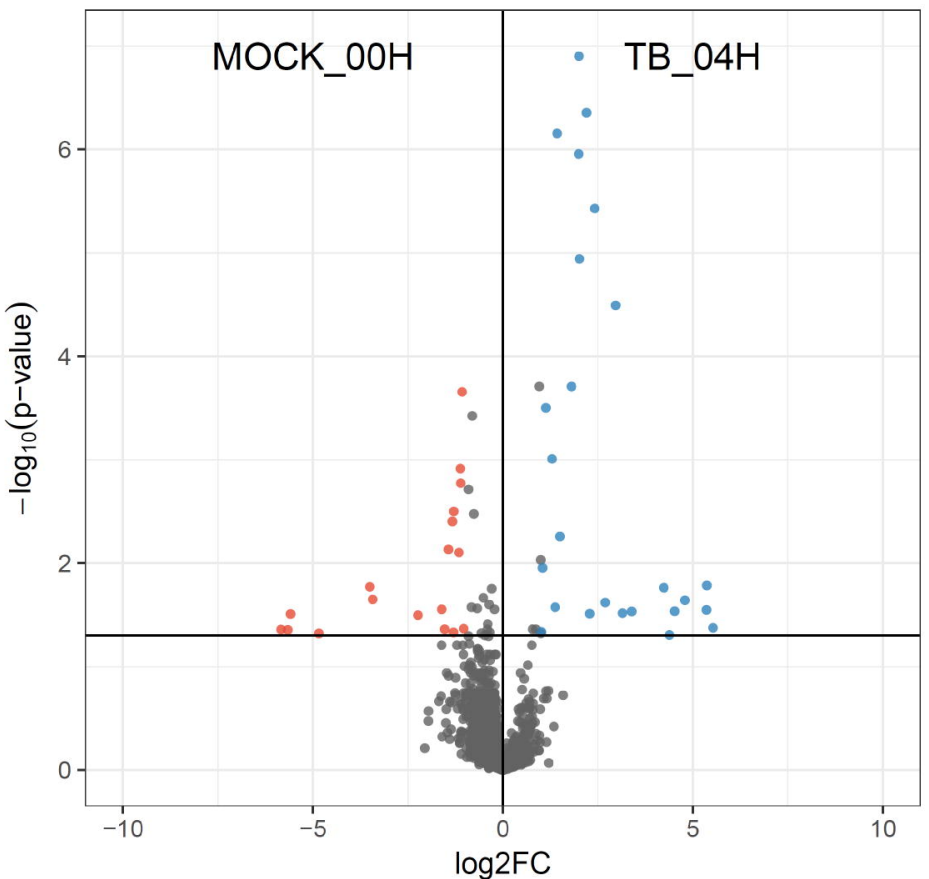

TB_24H-MOCK_24H

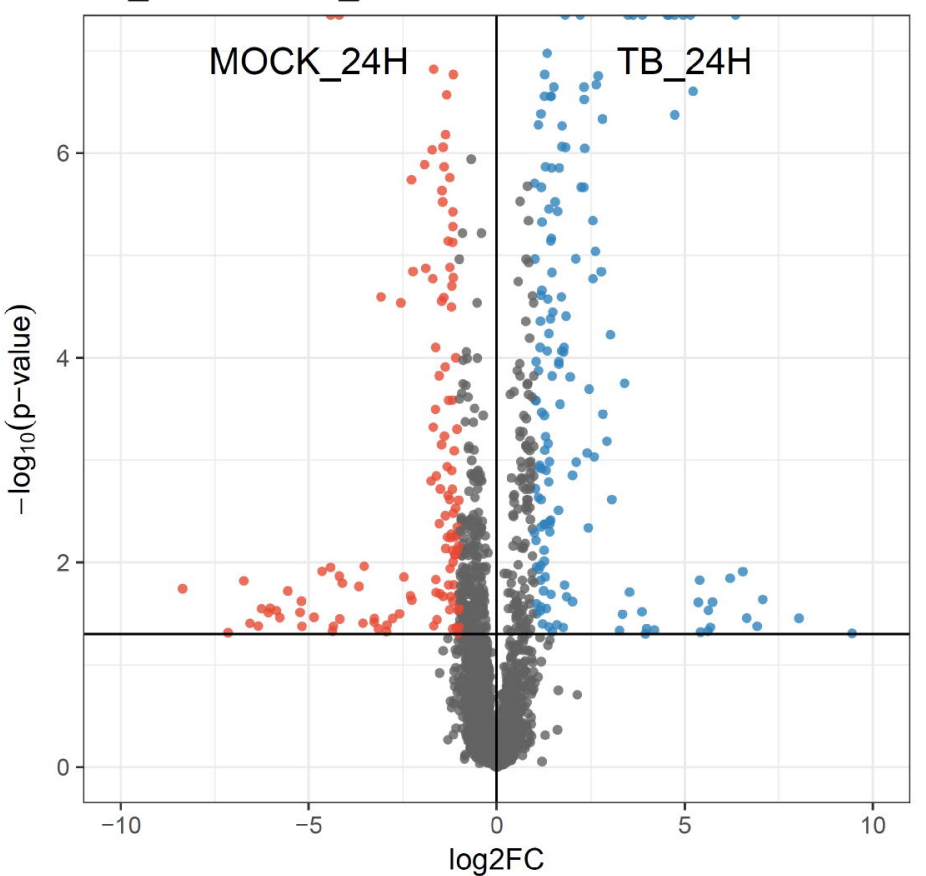

TB_06H-MOCK_06H

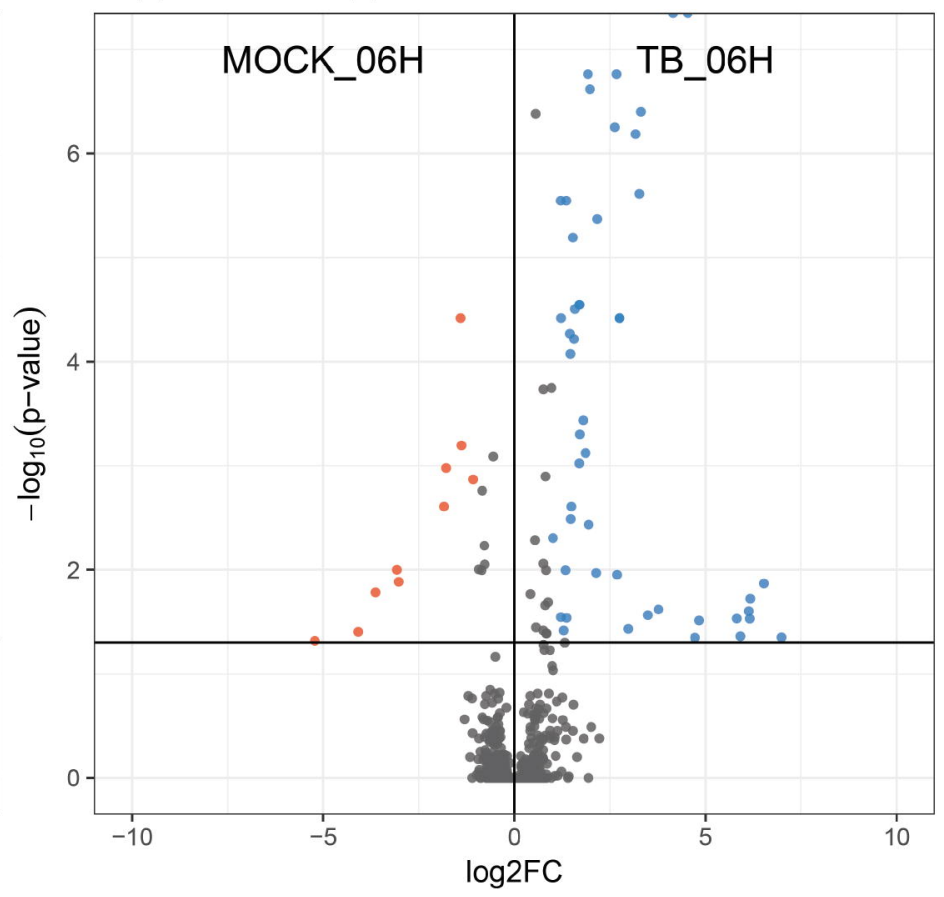


Fig. S3
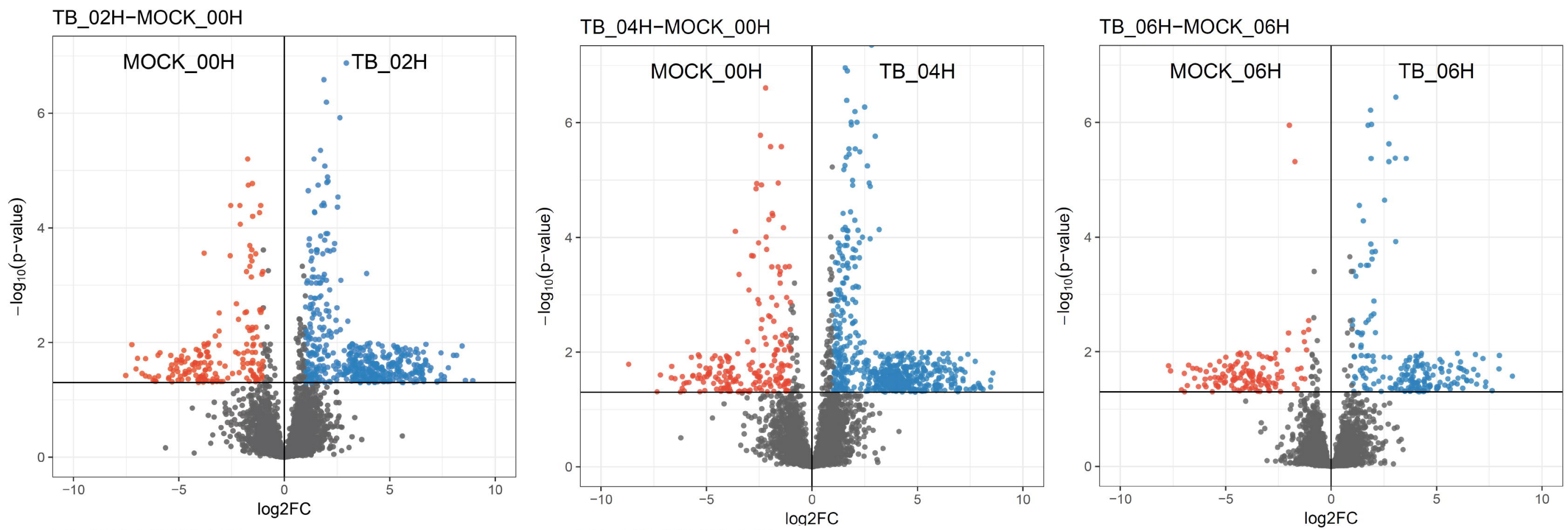

TB_08H-MOCK_06H

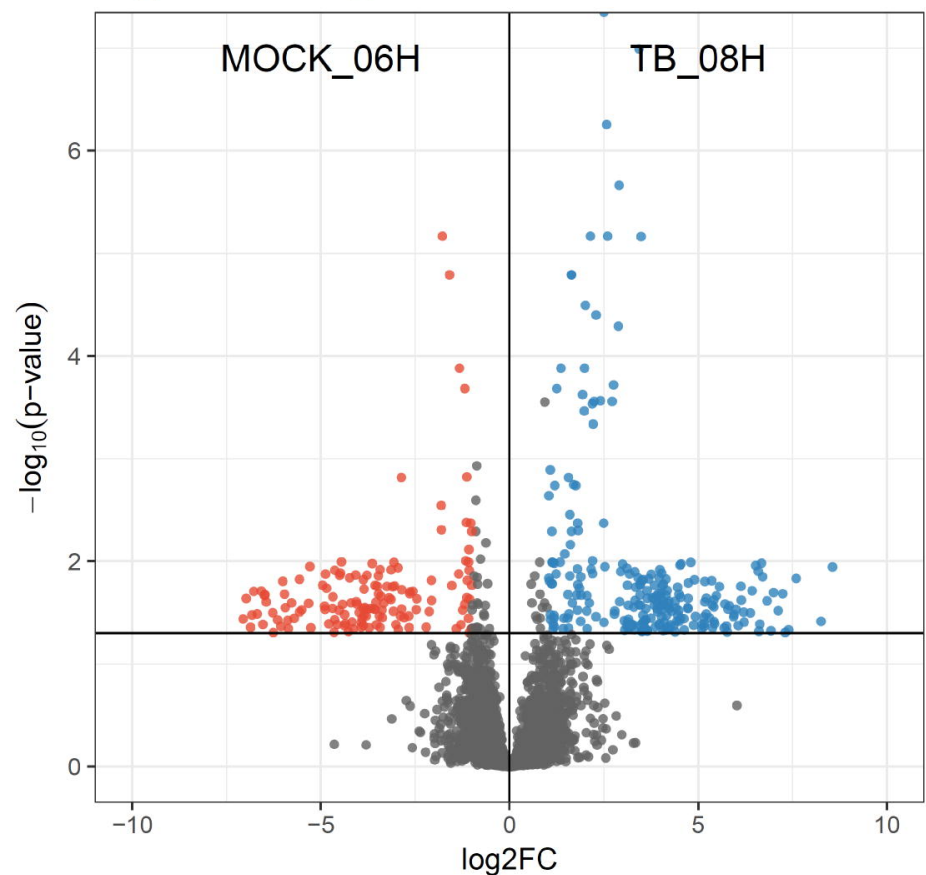


Fig. S5

2 hours post-infection
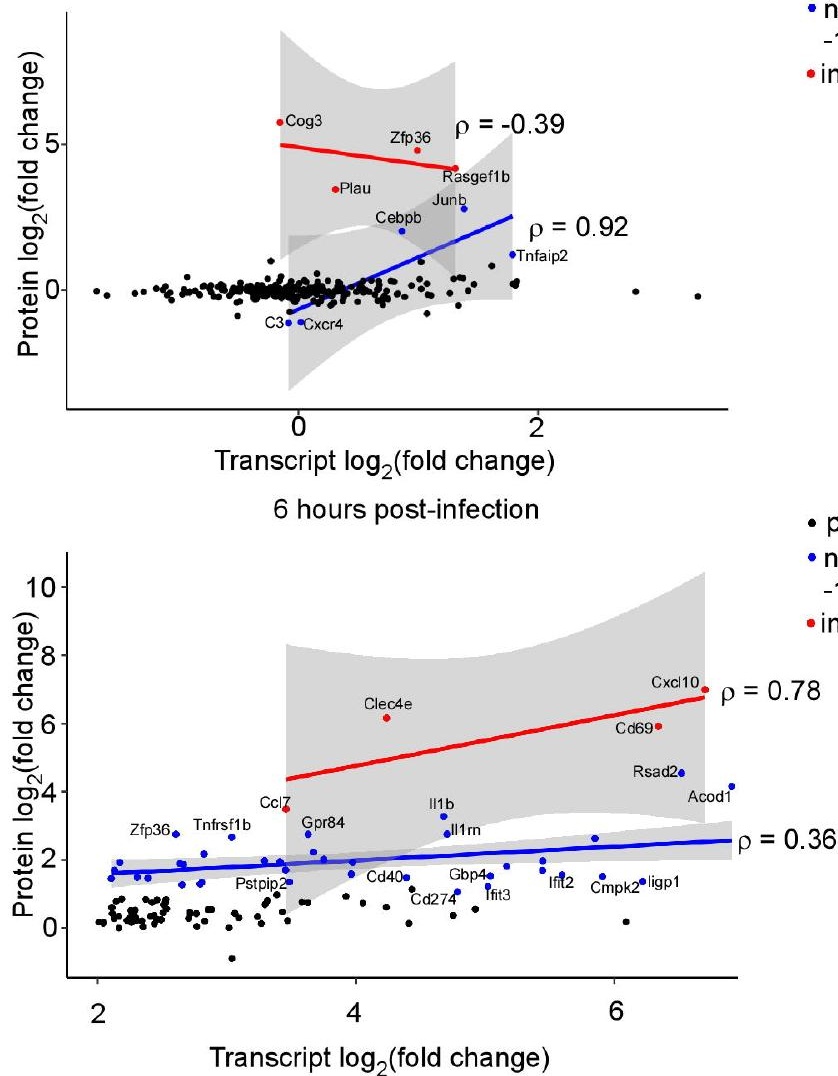

- proteins with no changes

- non-imputed proteins $-1>\log _{2}$ FC $>1, p<0.05$ - imputed proteins
- proteins with no changes - non-imputed proteins $-1>\log _{2} \mathrm{FC}>1, \mathrm{p}<0.05$ -imputed proteins 


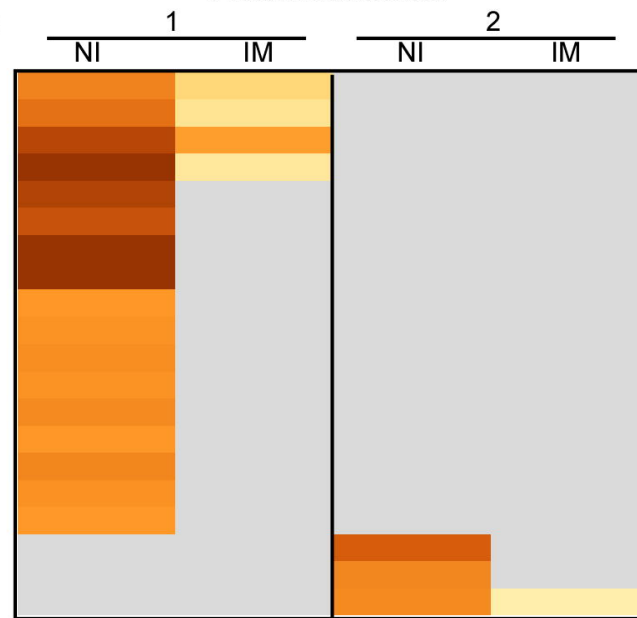

NI: Non-imputed IM: Imputed $-\log _{10}(p)$ $\begin{array}{llllll}0 & 3 & 6 & 10 & 20\end{array}$

hegative regulation of cytokine production

eukocyte cell-cell adhesion

response to bacterium

regulation of defense response

regulation of immune effector process

response to IFN-

response to IFN- $\beta$

response to virus

acute inflammatory response

response to type I IFN

regulation of response to biotic stimulus

regulation of phagocytosis

reactive oxygen species metabolic process

positive regulation of multi-organism process

regulation of production of molecular mediator of immune response apoptotic signaling pathway

NLRP3 inflammasome complex assembly

maturation of LSU-rRNA

regulation of protein catabolic process in the vacuole

DNA replication

movement/trafficking metabolism

signal transduction immunity other

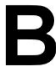

Class

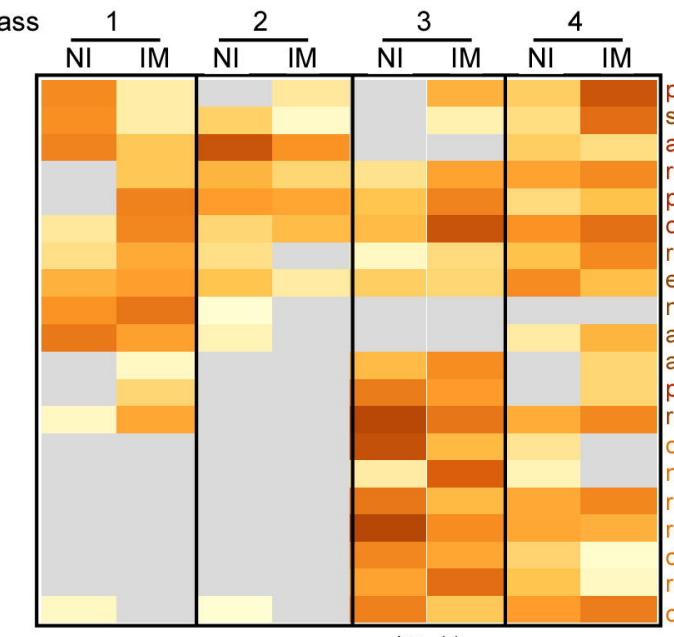

NI: non-imputed

IM: imputed

$-\log _{10}(p)$

C

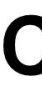

Clas

Phosphorylated Proteins

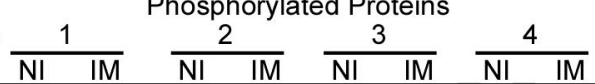

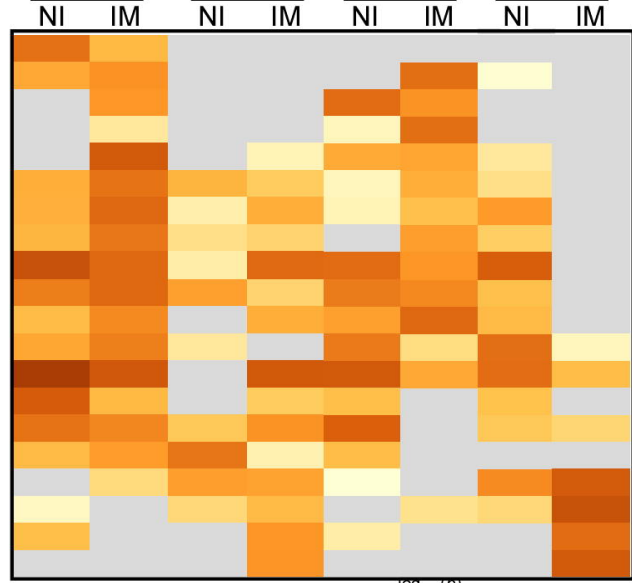

NI: non-imputed

100

IM: imputed purine-containing compound metabolic process

supramolecular fiber organization

amide biosynthetic process

regulation of proteolysis

protein modification by small protein conjugation

cellular protein catabolic process

regulation of cellular protein localization

endocytosis

nucleosome assembly

actin cytoskeleton organization

autophagy

positive regulator of catabolic process

regulation of multi-organism process

cellular response to IFN- $\beta$

negative regulation of defense response

response to IFN-r

regulation of innate immune response

cytokine production

regulation of immune effector process

cellular response to IFN- $\gamma$

\section{movement/trafficking metabolism signal transduction immunity}

early endosome to late endosome transport

transmembrane receptor tyrosine kinase signaling pathway

I-kB kinase/NF-kB signal transduction

regulation of IL-2 production

regulation of cell adhesion

cellular response to hormone stimulus

regulation of cell migration

response to peptide

small GTPase mediated signal transduction

regulation of catabolic process

regulation of GTPase activity

regulation of cell morphogenesis

actin cytoskeleton organization

protein-containing complex assembly

negative regulation of cellular component organization

protein localization to the plasma membrane

cellular response to DNA damage stimulus

regulation of chromosome organization

cell division

DNA replication

movement/trafficking metabolism signal transduction immunity other 


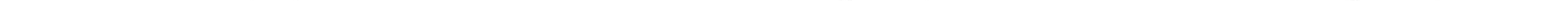




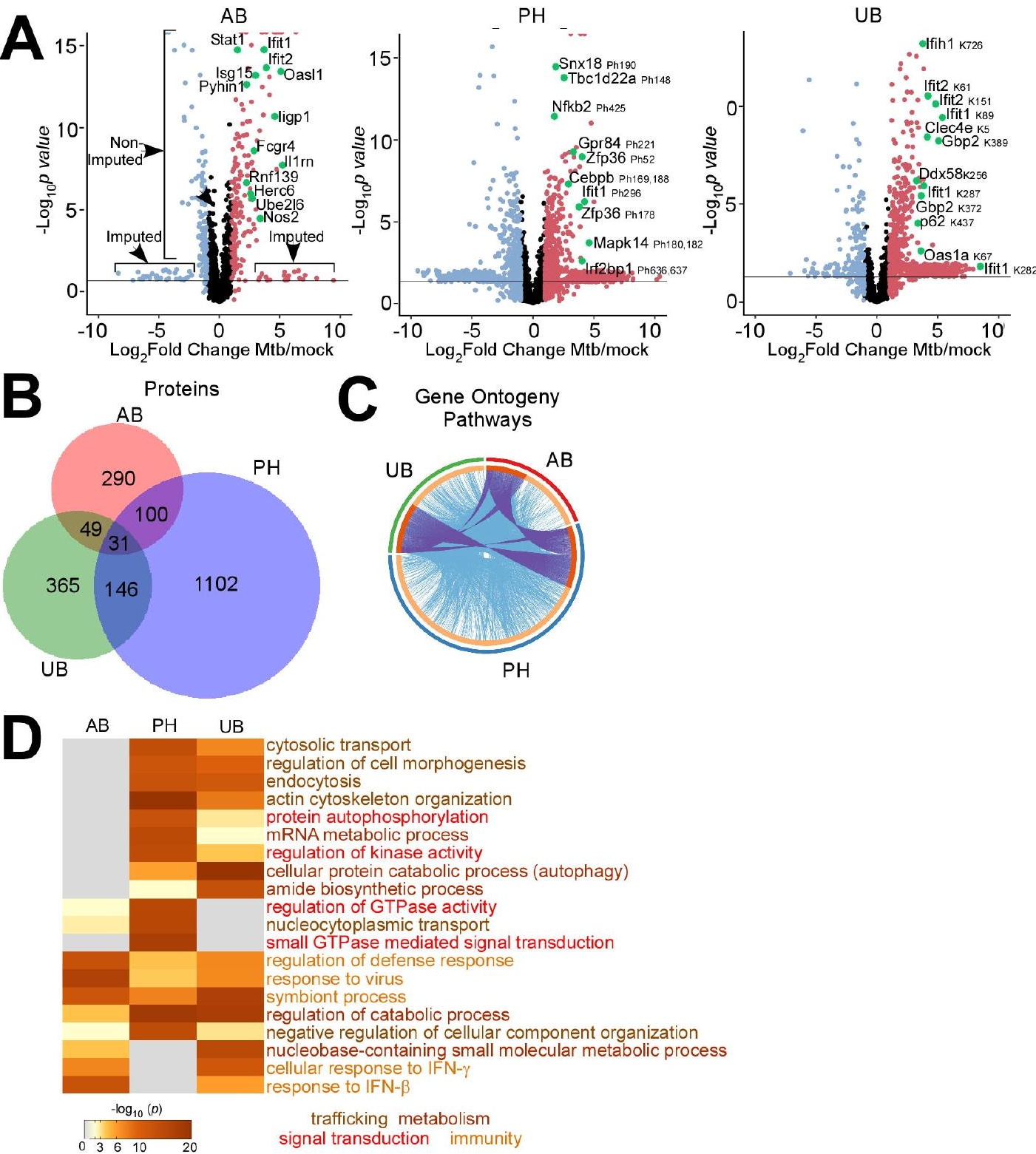


Ubiquitylated

Class

\begin{tabular}{lllll}
\hline 2 & 4 & 6 & 8 & 24
\end{tabular}

Representative Ubiquitylated Proteins

1

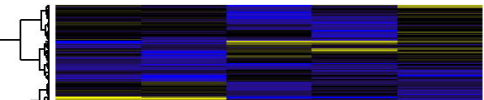
Actb, Arpc1b, Cdc42, Itgb5, Lcp1, Rac1, TIn1, Coro1b, Atxn3, Strip1

Amide biosynthetic process

3<smiles>C1C[C@@H]2CC3CC1CC2C3</smiles>

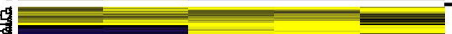

Eef2, Rpl13a, Rps3, Rpl11, Rps20, Rps9

Cellular protein catabolic process Apoe, |ltch, Sqstm1, Psmc3, Ripk1, Tnfaip3, Psma5, Tollip, Usp19, Rnf114, Trim25, Rnf213

4

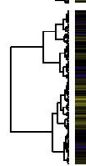

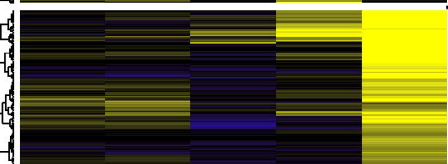

Purine-containing compound metabolic process Aldoa, Cap1, Gnai3, Hspa8, Pfkl, Pgk1, Tpi1, Oasl2, Samhd1, Ahcyl2, Vcp

Ubiquitylated Imputed Peptides $(n=641)$

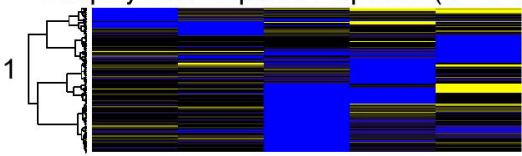

\section{Nucleosome assembly Hist1h1d, H2afy, Hist1h1e, Hist1h2bc, Hist1h3f, Hist4h4}

2<smiles>C1C2CC1C2</smiles><smiles>C1C2CC3CC1CC(C2)C3</smiles><smiles>C=C=[Ge]</smiles>

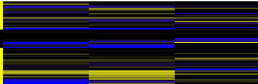

Amide biosynthetic process Eif3a, Rack1, Rpl18, Rpl32, Rpl7, Sptlc2 Rpl27a, Rpl7a, Rpl37, Nars, Rpl13

Regulation of innate immune response Cd14, Acod1, Trim30a, Tnfaip3, Tnip1, Ifih1, Ripk2, Ddx58, Parp14

\section{Endocytosis}

Ap2m1,Hck, Myh9, Rab11b, Vav1, Gapvd1, Ehd4, Plcg2, Fkbp15

$\log _{2}$ (fold change)

$$
<-3-2-1012>3
$$

\section{Ubiquitylated proteins}

Class

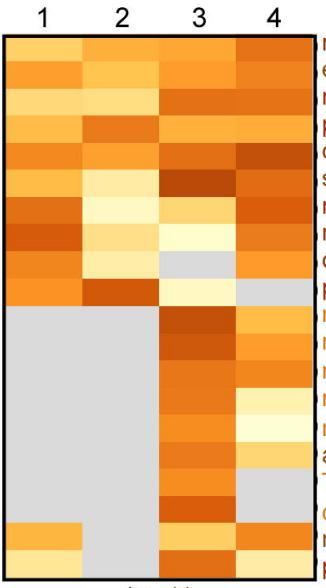

regulation of protein complex assembly endocytosis

regulation of cellular catabolic process

protein modification by small protein conjugation cellular protein catabolic process

symbiont process

nicotinamide nucleotide metabolic process

ribose phosphate metabolic process

cellular aldehyde metabolic process

peptide metabolic process

regulation of defense response

regulation of innate immune response

response to IFN-y

regulation of immune effector process

regulation of $\mathrm{I}-\mathrm{kB}$ kinase/NF-kB signaling

autophagy

T cell activation

cellular response to IFN- $\beta$

regulation of cellular protein localization

protein folding 
$\Delta$ Non-imputed Phosphopeptides $(\mathrm{n}=1705)$

\section{Hours Post-infection}

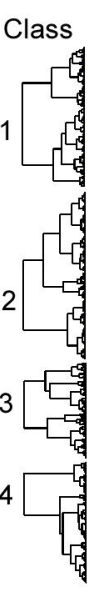

\section{Phosphorylated Proteins}

Actin cytoskeleton organization

Arpc1b, Cd2ap, Epb41|1, Evl|Inppl1, Lats1, Marcks, Pxn, TIn1, Lima1, Arhgap17, 3110043O21Rik, Arhgap12, Baiap2, Mical1, Bcas3, Mtss1, Mkl1, Pak2, Arhgap25, Kank2, Epb41

Actin cytoskeleton organization Abi1, Eps8, Ikbkb, Arhgef2, Pip5k1a, Pxn, TIn1, Sh2b2, Rufy3, Jmy, Lima1, Carmil1, Fmnl2, Baiap2, Bcas3, Sipa111, Ssh1, Iqsec1, Iqgap2

Protein localization to the plasma membrane Akt1, Fcer1g, Nsf, Gripap1, Fam126a, Scrib, Fcho2, Rab8b

Actin cytoskeleton organization

Abi1, Bcl6, Cfl1, Arhgef2, Lrp1, Mef2a, Sirpa, Sh3bp1, Mink1, Tsc1, Arhgap17, Arhgap12, Atxn3, Bcas3, Llgl2, Abi2

Imputed Phosphopeptides $(n=1253)$
2
(1)
Actin cytoskeleton organization
Arhgap6, Cfl1, Evl, Marcks, Prkar1a, TIn1, Sdcbp, Nisch, Spire1, Carmil1, Shtn1, Arhgap18, Clasp2, Flna, Sipa1I1, $\mathrm{Mk} 12$
Regulation of GTPase activity Srgap2, Plxnc1, Snx9, Plxnd1, Tbc1d5, Sh3bp4, Dock10, Eif5, Arhgap1, Rasal3
Small GTPase mediated signal transduction Bcl6, Eps8, Rock1, Trim28, Git2, Dlc1, Dock5, Arhgap18, Dock11, Ralgps2, Sipa1l1, Arhgap35, Abi2
DNA replication
Cdk1, Mcm4, Pola2, Rrm2, Atrx, Ccdc88a, Chtf18, Atad

\section{$\log _{2}$ (fold change) \\ $<-3-2-1 \quad 0 \quad 1 \quad 2>3$}

\section{Phosphorylated proteins}

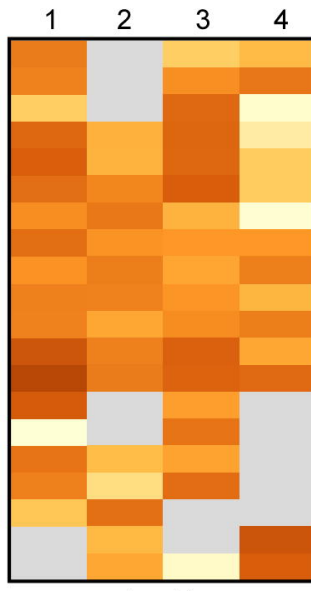

$-\log _{10}(p)$

regulation of intracellular transport regulation of cell morphogenesis I-kB kinase/NF-kB signal transduction regulation of cellular catabolic process regulation of catabolic process regulation of GTPase activity endomembrane system organization negative regulation of cellular component organization regulation of cellular protein localization protein localization to the nucleus positive regulator of cellular protein localization small GTPase mediated signal transduction actin cytoskeleton organization

cytosolic transport

regulation of IL-2 production

import into cell endosomal transport protein-containing complex disassembly regulation of DNA metabolic process regulation of chromosome organization 

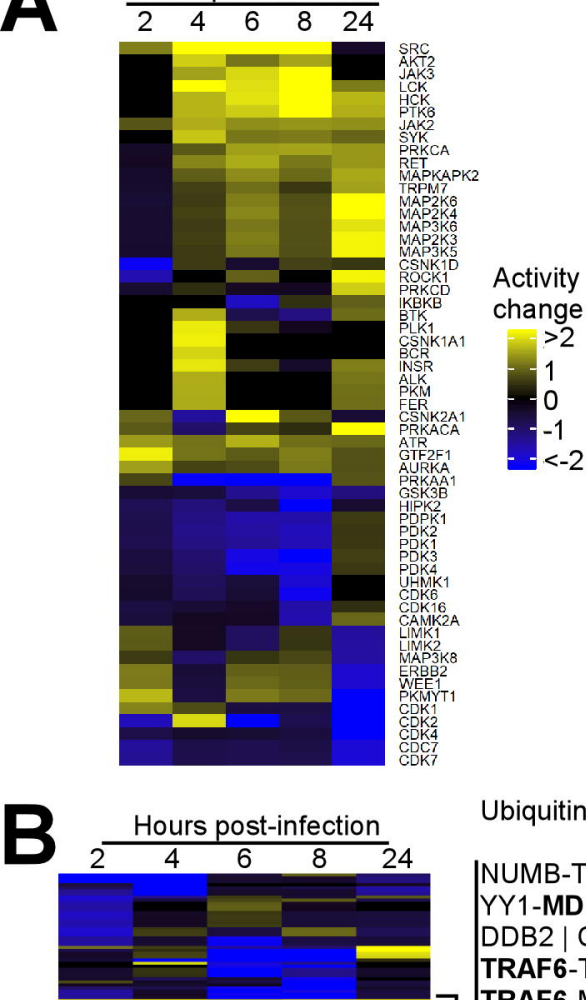

Ubiquitin ligase complexes

NUMB-T53-MDM2

YY1-MDM2-p53

DDB2 | CSA

TRAF6-TAK1

7 TRAF6-MALT1

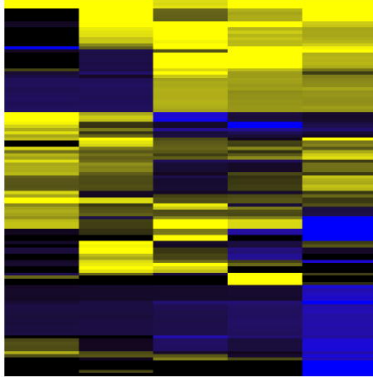

TRAF6-MALT1-UEV1A-BCL10 INIPA-SKP1A-CUL1-RBX1 SPOP-DAXX-CUL3 SPOP-DAXX-RBX1

Activity $<-2-1 \quad 0 \quad 1>2$ change 


\begin{tabular}{|c|c|c|c|c|c|}
\cline { 2 - 6 } \multicolumn{1}{c|}{} & \multicolumn{5}{c|}{ Phosphorylation } \\
\cline { 2 - 6 } \multicolumn{1}{c|}{ Hrs. post-infection } \\
\hline AP receptor & 2 & 4 & 6 & 8 & 24 \\
\hline p62 & $4^{*}$ & $3^{*}$ & $7^{*}$ & $8^{*}$ & $6^{*}$ \\
\hline BNIP3 & 0 & 0 & 0 & 0 & 0 \\
\hline BNIP3L & 5 & 5 & $6^{*}$ & 7 & 7 \\
\hline FUNDC1 & 2 & 2 & 3 & 3 & 3 \\
\hline NBR1 & 0 & 0 & 0 & 0 & 0 \\
\hline NDP52 & 0 & 0 & 0 & 0 & 0 \\
\hline Optineurin & $5^{*}$ & $7^{*}$ & $5^{*}$ & $6^{*}$ & $3^{*}$ \\
\hline TAX1BP1 & 0 & 0 & $1^{*}$ & $1^{*}$ & 0 \\
\hline
\end{tabular}
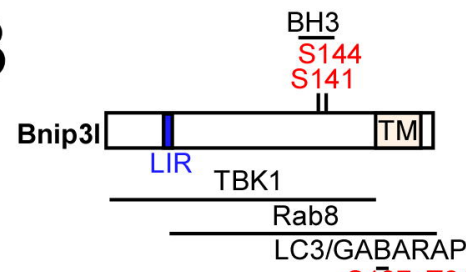

S187 T212
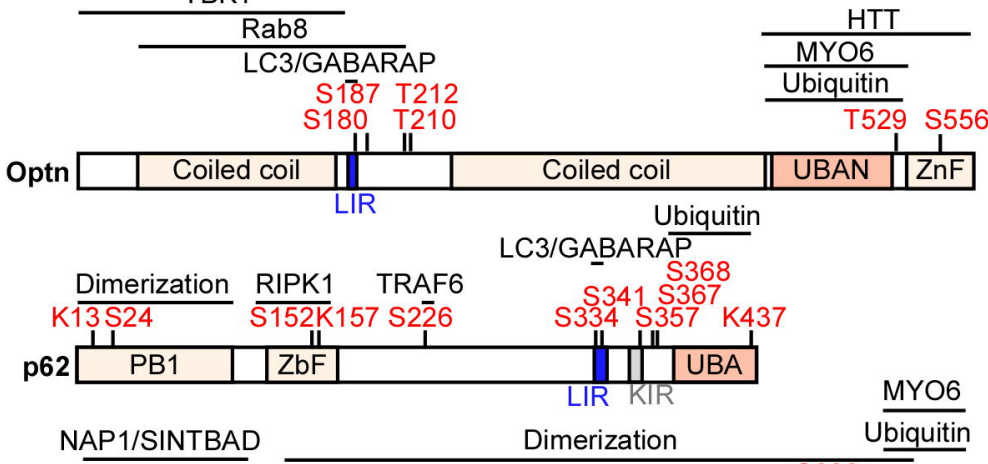

NAP1/SINTBAD

Dimerization Ubiquitin
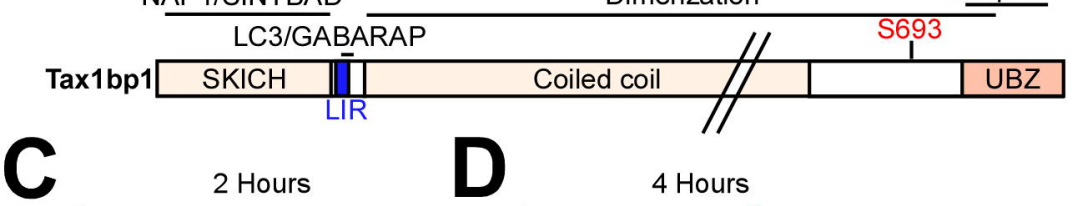

\section{Hours}

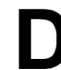

4 Hours
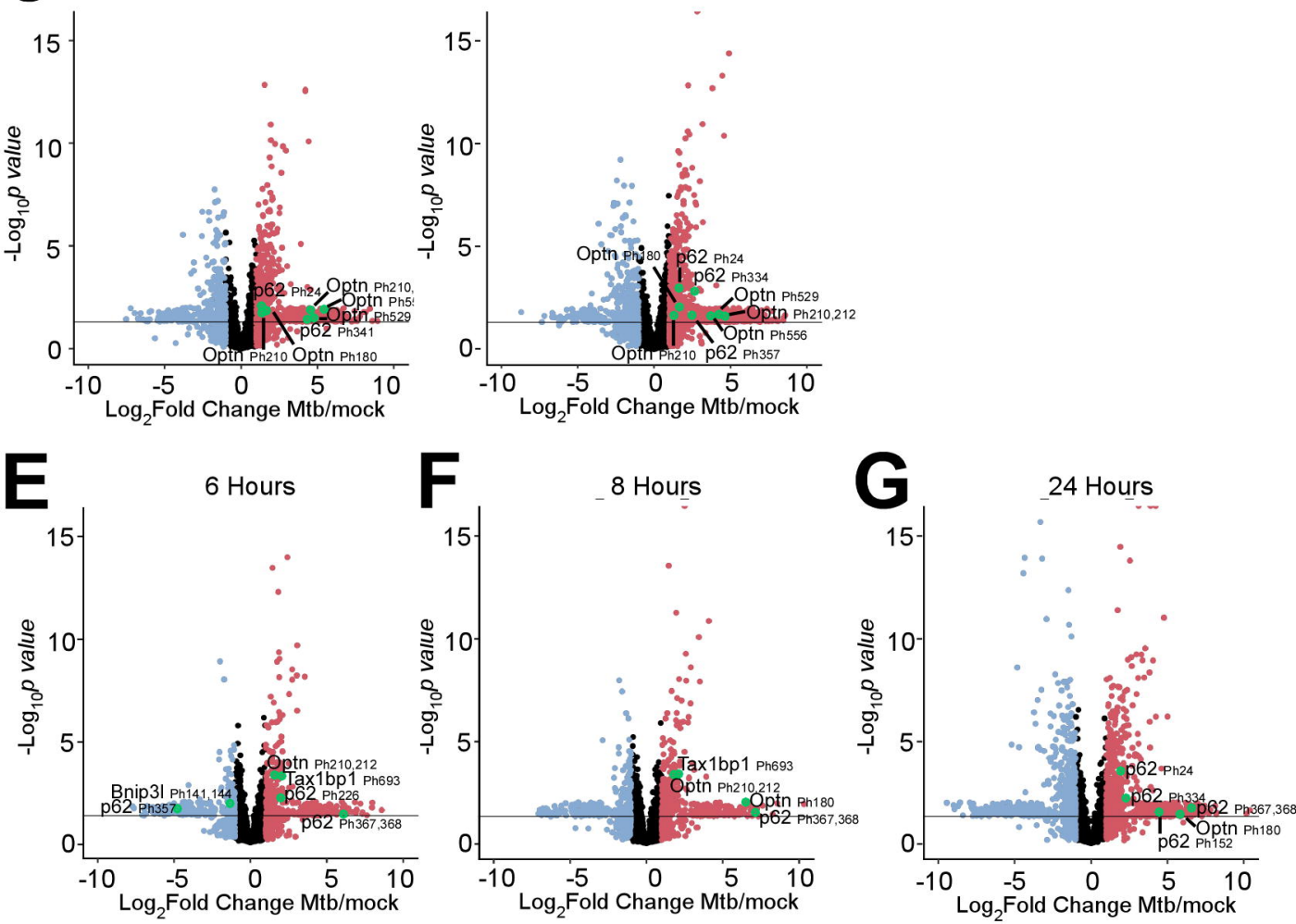
Fig. 8

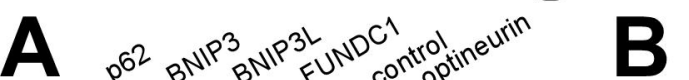

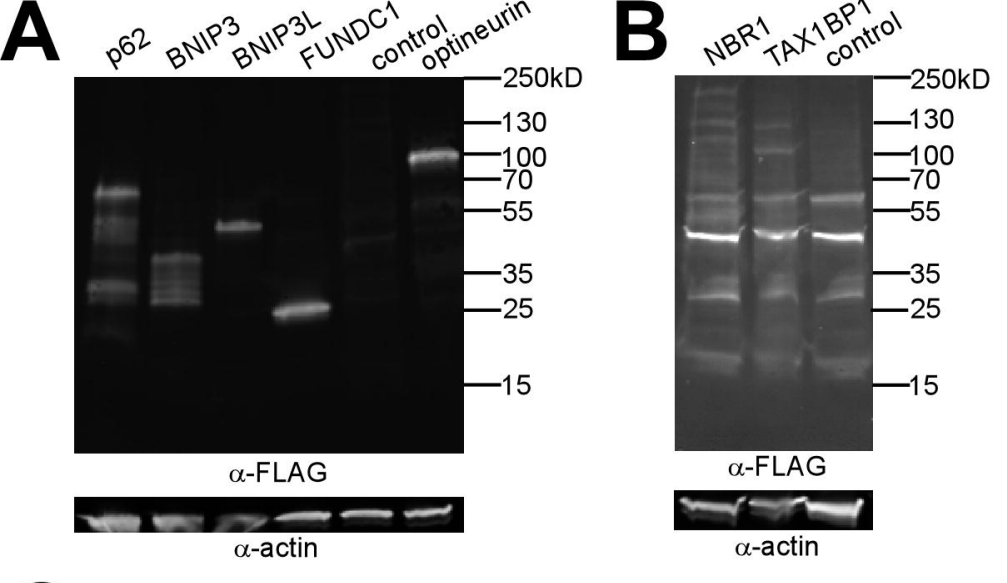

C

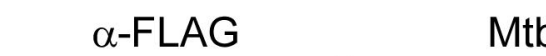

merge

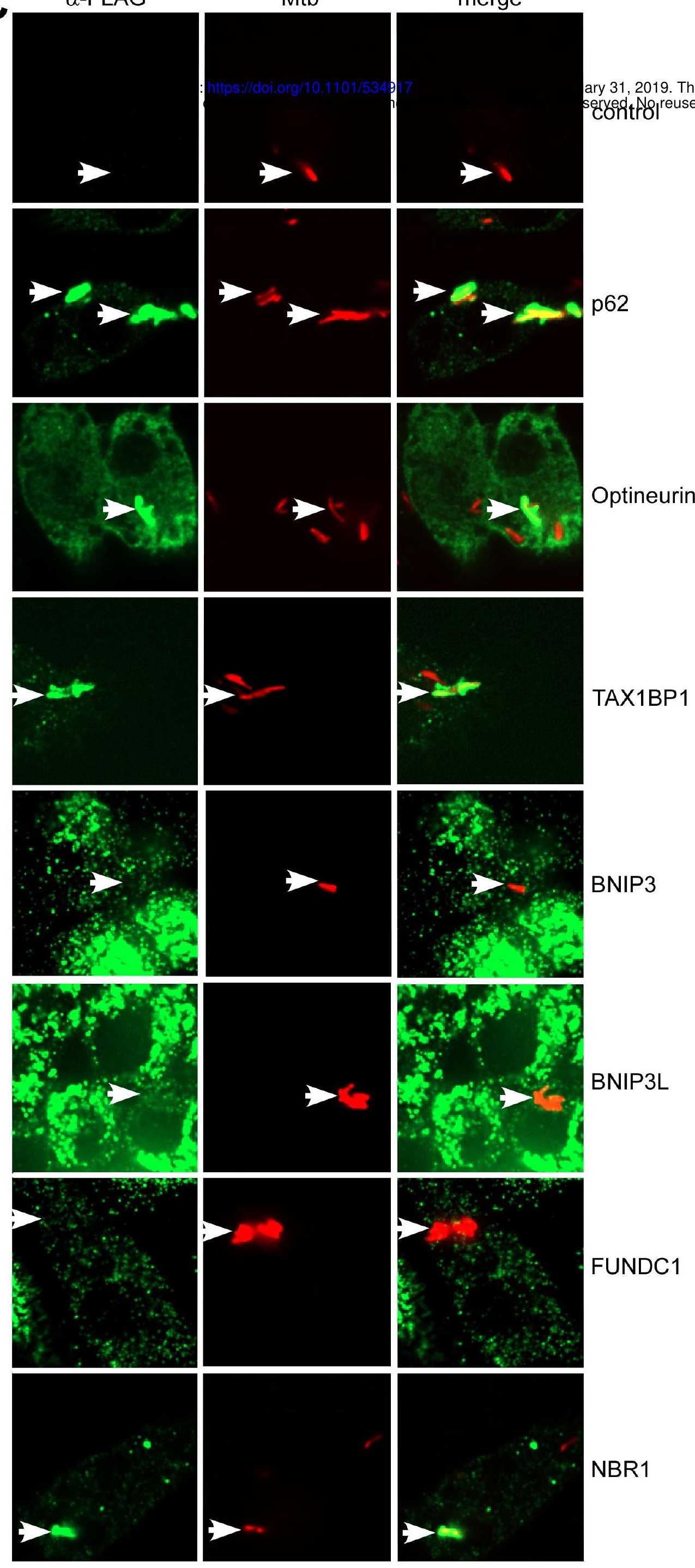


Fig. 9

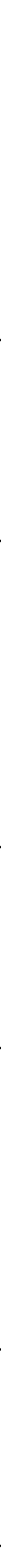

B

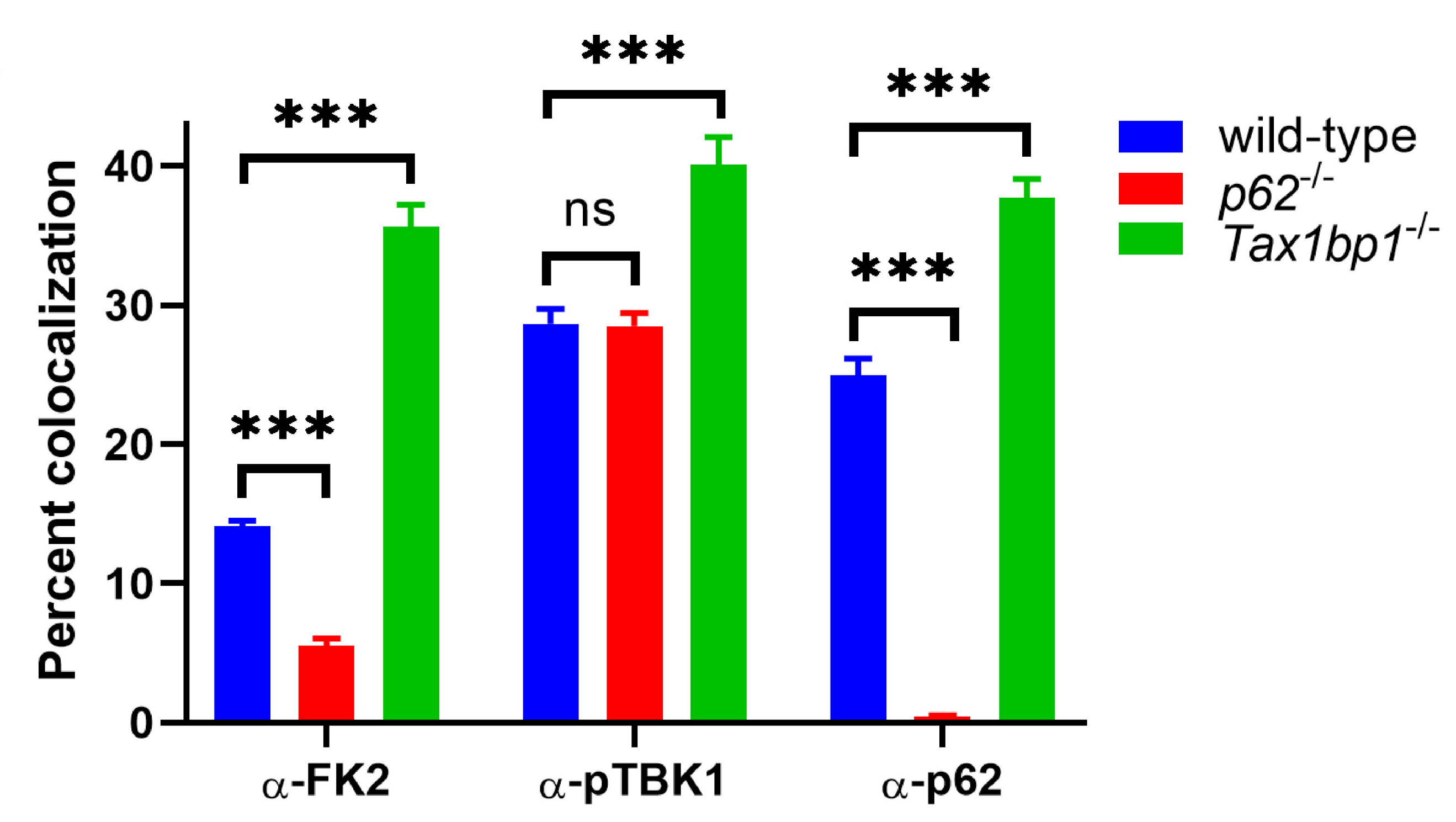




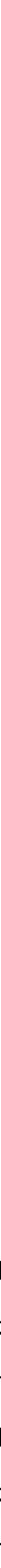

B

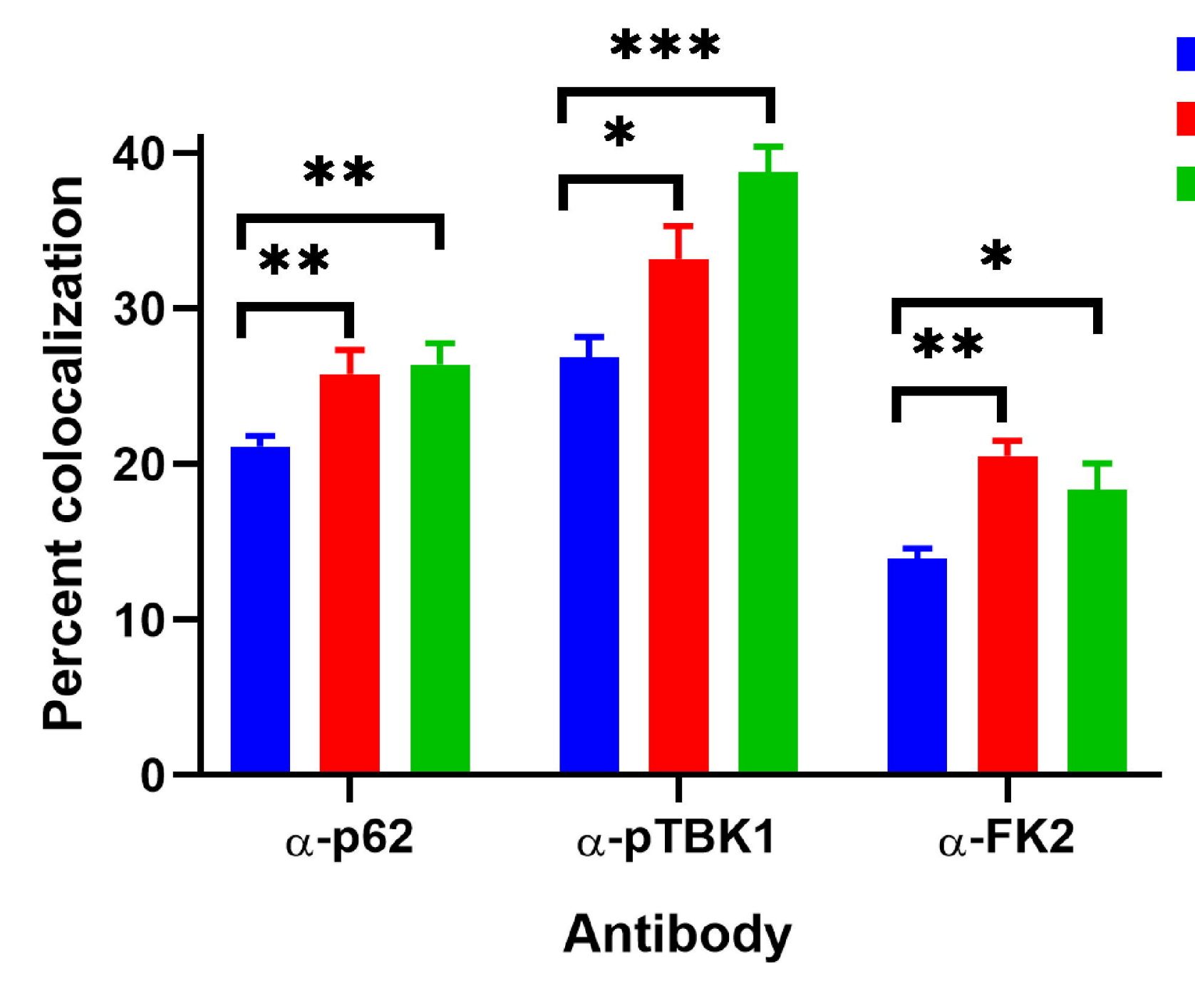

- Scramble controls

- Tax $1 b p 1$ exon 10

Tax1bp1 exon 9 
Fig. 11

A $\quad \alpha-L C 3$

Mtb

DAPI

merge

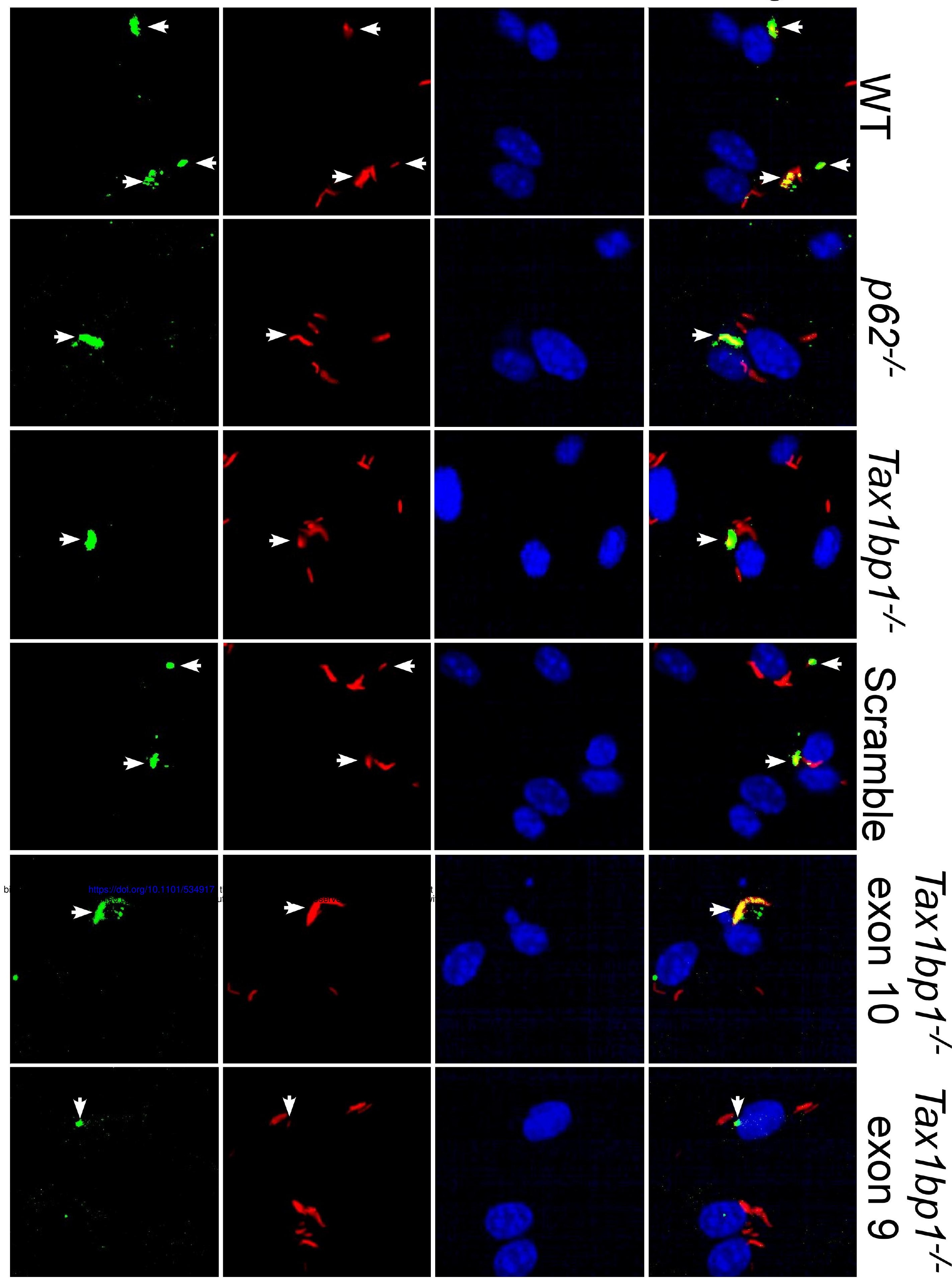

B

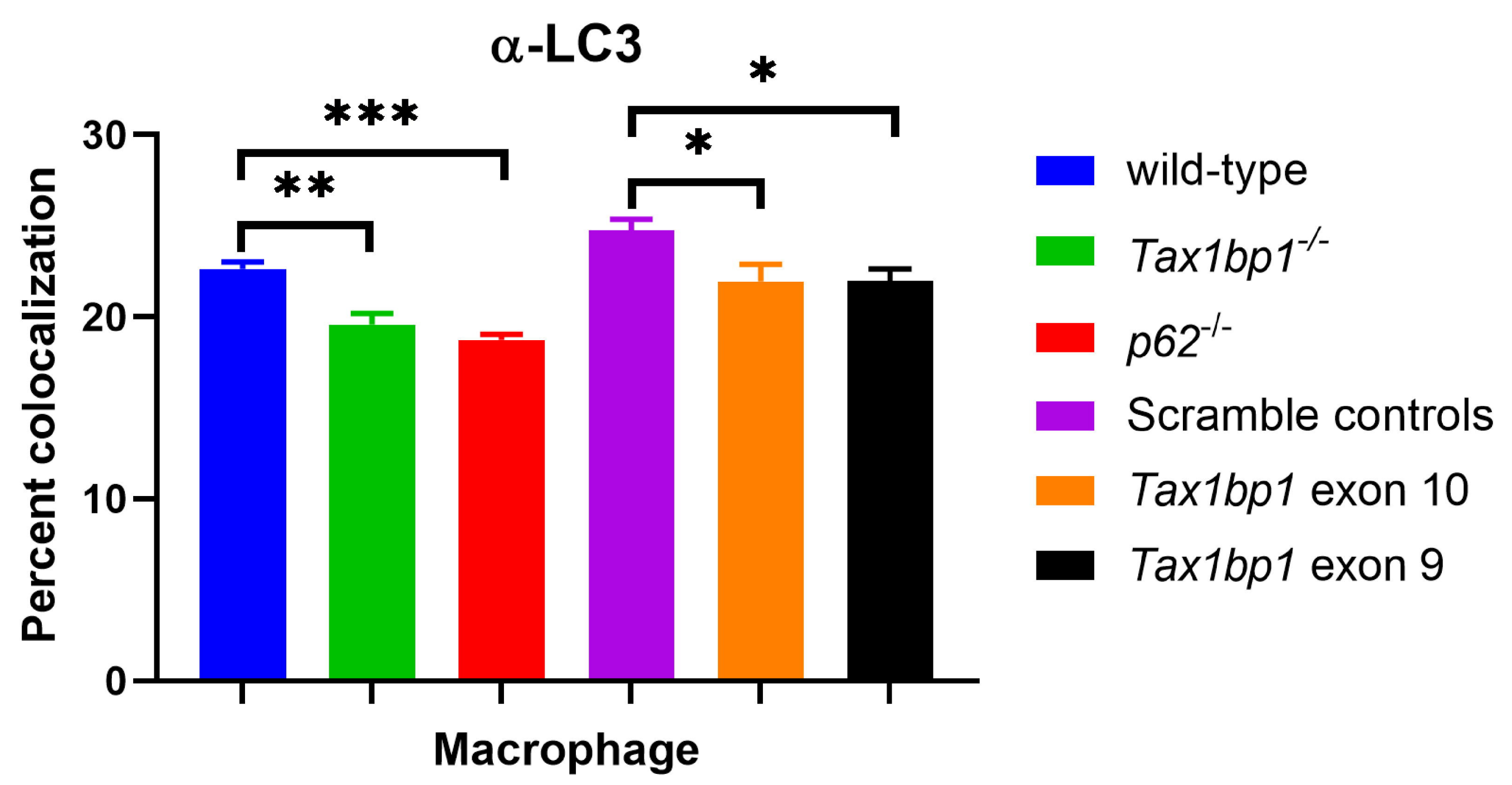




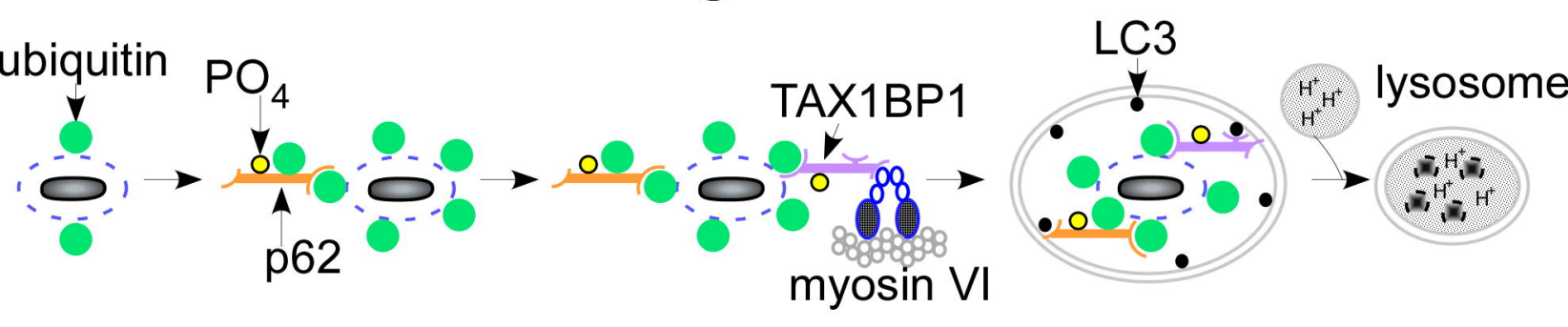

